\title{
Estimación y comparación de la competitividad regional en Colombia
}

\section{Departmental regional competitiveness rates in Colombia}

Elcira Solano-Benavides ${ }^{1}$

\author{
Profesora investigadora de la Universidad del Atlántico, Barranquilla, Colombia \\ elcirasolano@hotmail.com \\ https://orcid.org/0000-0001-6405-5359
}

Nelson De Jesús Alandete-Brochero ${ }^{2}$

Investigador de la Universidad del Atlántico, Barranquilla, Colombia alandete@outlook.es

https://orcid.org/0000-0001-9438-8795

Recibido: 04-12-18

Aprobado: 27-08-19

1 Doctora en Ciencias Económicas.

2 Economista. 


\section{Resumen}

El objetivo de esta investigación es estimar la competitividad regional en Colombia, analizarla según teorías económicas y establecer su relación con el PIB departamental. Se emplean metodologías cuantitativas, análisis de componentes principales y modelos de datos de panel. Entre los principales resultados se encontraron siete índices de competitividad relacionados con la teoría económica: el de aglomeración tiene un alto grado de desigualdad con el resto de departamentos; el de fuerza centrífuga está relacionado con los factores de la tierra y la minería; el de infraestructura presenta grandes brechas; el de capital humano es el que presenta menor desigualdad por la coberturas en educación en los departamentos; el de economía geográfica revela, igualmente, baja desigualdad mostrando que los costos de transporte han disminuido en Colombia; el de economía institucional es el que presenta menor desigualdad entre los departamentos y por último el de ciencia, tecnología e innovación muestra una gran desigualdad.

Palabras clave: competitividad; geografía económica; capital humano; componentes principales; aglomeración. Clasificación JEL: D31; R11; C23; C38; R12.

\section{Abstrac}

This research aims to analyze the regional departmental competitiveness in relation to economic theories and regional per capita GDP in Colombia. A quantitative methodology of principal component analysis and panel data models is used. Among the main results, seven competitiveness indices related to economic theory were found: that of agglomeration has a high degree of inequality with the other departments; that of centrifugal force is related to the factors of land and mining; the infrastructure has large gaps; that of human capital is the one that presents the least inequality due to the coverage in education in the departments; that of geographic economy also reveals low inequality, showing that transportation costs have decreased in Colombia; the one of institutional economy is the one that presents less inequality between the departments and finally the one of science, technology and innovation shows great levels of inequality.

Key words: Competitiveness; Economic Geography; Human Capital; Principal Components; Agglomeration.

¿Cómo citar este artículo? / How to quote this article?

Solano-Benavides, E. y Alandete-Brochero, N. J. (2020). Estimación y comparación de la competitividad regional en Colombia. Sociedad y economía, (39), 80-112. https://doi.org/10.25100/sye.voi39.8634 


\section{Introducción}

La competitividad regional es un proceso de generación de competencias, el cual depende de la capacidad de crear un entorno que favorezca el crecimiento sostenido de la productividad y el nivel de vida de la población, considerando las capacidades que ofrece el territorio para facilitar el desarrollo y la actividad económica (Porter, 1990; Begg, 2002; Cabrero, Orihuela y Ziccardi, 2003; Musik y Romo, 2004).

En tal sentido, la teoría económica ha desarrollado el tema de la competitividad desde distintas teorías. Por ejemplo, la escuela clásica con la teoría de ventajas absolutas (Smith, 1776), la teoría de las ventajas comparativas (Ricardo, 1817) y el modelo de Heckscher-Ohlin (Hecksher, 1919; Ohlin, 1933). Sin embargo, es la teoría de aglomeración la que tiene el mayor aporte en los estudios de competitividad; las regiones más competitivas tienen aglomeración económica y demográfica que permite una disminución de costos de transportes, de transacción y laborales (Strange, 2005).

En Colombia los principales estudios sobre indicadores de competitividad regional son los elaborado por la Comisión Económica para América Latina y el Caribe (CEPAL) 2000-2015 y el Consejo Privado de Competitividad (CPC) 2012-2015. No obstante, el marco teórico no se identifica claramente debido a la gran cantidad de variables que presentan los indicadores, creando dificultad en la interpretación de los resultados y en la toma de decisiones de política económica (Lotero, Posada y Valderrama, 2009). Además, utilizan numerosos factores y variables, que no son los mismos en todos los años, como en el caso de la CEPAL, o utilizan metodologías cuantitativas subjetivas al determinar el peso de ponderación de los factores de competitividad, como en el caso de la CPC. La importancia de que los índices de competitividad estén fundamentados en la teoría económica radica en la información que proveen los índices y en que estos ayuden a proponer políticas económicas en términos de las potencialidades con las que cuenta cada región.
Reconociendo que existe un problema en cuanto al marco teórico económico de los indicadores de competitividad estimados en Colombia, es necesario formular la siguiente pregunta: ¿Los indicadores regionales de competitividad departamental estimados en esta investigación se encuentran fundamentados en las teorías económicas?

El objetivo de esta investigación es estimar y analizar un indicador de competitividad regional departamental que esté relacionado con las teorías económicas y con el PIB per cápita de los departamentos en Colombia.

La metodología utilizada en el trabajo es cuantitativa; inicialmente se evalúan los indicadores regionales departamentales de la CEPAL y del CPC calculados para Colombia. Además, se estiman los indicadores de competitividad propuestos en este trabajo con el análisis factorial de componentes principales; e igualmente se utiliza un modelo econométrico de panel data para evaluar los indicadores de competitividad regional con el PIB per cápita departamental.

Así mismo, la hipótesis planteada es que los indicadores regionales de competitividad departamental están enmarcados con las teorías de aglomeración, economía geográfica, economía institucional y capital humano.

El presente trabajo está organizado de la siguiente manera: en la primera parte se presentan las discusiones teóricas del concepto de competitividad regional. En la segunda se evalúan los índices de competitividad departamental de la CEPAL y del Consejo Privado de Competitividad. En la tercera se estiman y analizan los indicadores de competitividad regional departamental fundamentados con la teoría económica. En la cuarta, se evalúan los indicadores regionales de competitividad estimados con la productividad departamental. Por último, la discusión y conclusión de los resultados.

\section{Discusión teórica}

Los grandes precursores del tema de competitividad provienen de la escuela clásica y su interés por el comercio exterior. Fue Adam 
Smith(1776)quieninicialmenteexpusolosfactores que permiten la especialización y aumento de la productividad, por ello consideraba que la inversión en capital y el aumento del tamaño del mercado se ven reflejados en el comercio y, por lo tanto, cada país se especializa en producir un bien a un costo absolutamente menor que otro. Por su parte, David Ricardo (1817) demostró que las ganancias del comercio resultan cuando dos países se especializan en la producción de bienes para los que poseen una ventaja comparativa. Del mismo modo, la escuela neoclásica amplía el modelo de ventajas comparativas con el modelo Heckscher-Ohlin (Martin, 2003); el cual afirma que los países se deben especializar en el comercio internacional de bienes y servicios que se produzcan con factores abundantes de cada país (Martin, 2003).

Para Krugman (1995) es importante distinguir la competencia empresarial con la de un país o región, pues la competencia de los países en el comercio mundial no es igual a la que pueda tener un grupo de empresas, el éxito o fracaso de la economía de un país está en función de sus propios esfuerzos y no de las pérdidas que pueda tener otra nación, es decir, las competencias entre regiones no se comportan como un juego de suma cero. Entre los países y/o regiones no existe una competencia como entre las empresas, más bien se da una complementariedad, una relación y no un enfrentamiento en el comercio internacional o interregional (Lotero et al., 2009).

Es así que las diferencias regionales son producto de la aglomeración o concentración espacial de la actividad económica en regiones determinadas, creando ventajas en términos de eficiencia para desempeñar las diferentes actividades de la sociedad, generando a su vez economías de escala (Strange, 2005; Manrique, 2006). Adicionalmente, la relación sobre los factores escasos y abundantes es explicada por la complementariedad económica, la cual consiste en intercambiar productos de diferentes industrias, especializándose cada región en los productos que tienen ventaja comparativa. Es así que Lotero et al. (2009), consideran que las regiones atraen recursos escasos y expulsan los abundantes; lográndose así ganancias de bienestar, convergiendo a un mismo nivel de ingreso per cápita. En esta situación las ganancias se obtienen por la complementariedad en el uso de los recursos y no por su acumulación.

Existen unos factores que logran facilitar el proceso, son los costos de transportes, comunicaciones y recursos complementarios que ayudan, como son: capital humano, ciencia, innovación y tecnología. Lo anterior produce en la región economías de escala, es decir, una demanda necesita ser abastecida y son necesarias plantas que satisfagan tal necesidad, lo cual conlleva a que se generen aglomeraciones.

El concepto de aglomeración es determinante para analizar la competitividad regional, en el sentido de que abarca beneficios de urbanización derivados de la presencia de varios actores y sectores en la misma área geográfica, además se fomenta la localización de empresas en el mismo lugar, generando la industrialización (Huovari, Kangasharju y Alanen, 2001). Basándonos en la teoría de Krugman (1988; 1997) de la nueva geografía económica, para las industrias existen incentivos y desincentivos para aglomerarse en una región determinada. Fujita et al. (1999) explican que a través de la causalidad circular se dan relaciones hacia adelante, donde las personas se concentran en regiones en las que se encuentran los productores y consumidores de bienes de consumo que ofrezcan mejores oportunidades de trabajo, formando así relaciones llamadas fuerzas centrípetas. Las fuerzas centrifugas son las que se dan por factores inmóviles como los recursos naturales, la minería, la agricultura y las fuentes de energía renovable.

Tales fuerzas pueden incentivar la dispersión de la actividad industrial de una región a otra. Según Fujita, Krugman y Venables (2014):

(...) la primera idea es que, en un mundo donde el aumento de la rentabilidad y los costes de transporte son importantes, eslabonamientos hacia adelante y hacia atrás pueden crear una lógica circular de aglomeración. Es decir, si todo 
lo demás se mantiene igual, los productores desearán localizarse cerca de sus proveedores y sus clientes, lo cual explica que van a terminar estando cerca los unos de los otros. La segunda idea es que la inmovilidad de algunos recursos -la tierra, ciertamente, y en algunos casos la fuerza laboral- actúa como una fuerza centrífuga que se opone a la fuerza centrípeta de la aglomeración. Y la tensión entre estas fuerzas centrífugas y centrípetas forman la evolución de la estructura espacial de la economía (...) (p. 345).

La teoría geográfica económica también aporta al concepto de competitividad regional. Este explica que la ubicación geográfica de la región afecta los costes de transporte en los mercados internos y externos, en el sentido de que las empresas siempre tratarán de minimizar costos y ubicarse en lugares donde existe mayor concentración de mercados (Fujita et al., 1999). Por otro lado, Belderbos y Carree (2002) exponen que las industrias centradas en la producción para la exportación tienen más probabilidades de establecerse en regiones con puertos marítimos que en regiones con concentración de consumo local. Así mismo, las condiciones extremas geográficas afectan la actividad económica de las regiones y el bienestar de los individuos que las habitan; Sánchez y Núñez (2000), encuentran que la cercanía a los mercados domésticos, las precipitaciones, la calidad del suelo y otros factores geográficos explican entre el $36 \%$ y el $47 \%$ de la variación en la renta municipal en Colombia.

También, la teoría del capital humano contribuye al concepto de competitividad regional para el crecimiento económico y la productividad en las regiones. Esto gracias a la generación de innovación que concibe nuevos productos o ideas determinantes para el progreso tecnológico (Romer, 1986). De tal manera, las regiones con mayores niveles iniciales de capital humano refuerzan la productividad del capital físico y conducen a la economía a un crecimiento sostenido, que funciona como un mecanismo endógeno para acelerar el proceso de crecimiento (Lucas, 1988).
Por otro lado, la nueva teoría institucional explica que los costes de transacción, confianza entre los actores económicos, corrupción, transparencia, gobierno abierto, capacidad empresarial, fortalecimiento de las redes y la cooperación entre los actores influyen en el desarrollo de las economías (Vázquez, 2000; Botero, 2011; Newman y Ángel, 2017). Así mismo, es necesario que existan instituciones que faciliten o disminuyan los costos de transacción, debido a que los altos niveles de corrupción y malos manejos públicos hacen menos competitivas a las regiones (Martin, 2003).

Además, la competitividad está relacionada con el ambiente empresarial que posee una economía o una región, tal como lo explica la teoría de clusters de Porter (1998). El autor indica que la concentración geográfica de empresas especializadas mejora la eficacia operativa de sus actividades y mantiene posiciones estratégicas que las diferencian. Sin embargo, la competitividad empresarial y la competitividad regional no podrán darse si no existe infraestructura económica óptima que produzca un círculo virtuoso urbanístico (Cohen, 2006).

\section{Evaluación de los indicadores de competitividad de la CEPAL y del Consejo Privado de Competitividad}

Los principales estudios de indicadores de competitividad departamentales en Colombia son los elaborados por la CEPAL (Ramírez y Parra, 2009; 2010; Ramírez, Osorio y Parra, 2007; Ramírez, Parra, González y Corredor, 2014; Ramírez y de Aguas, 2015) y por el Consejo Privado de Competitividad (CPC, 2013; 2014; 2015; 2016). El primero estima y analiza los factores de competitividad en los departamentos de Colombia, usando como metodología un modelo factorial por componentes principales y 71 variables agrupadas en cinco factores ${ }^{3}$. El segundo calcula indicadores de competitividad

3 Fortaleza Económica, Infraestructura, Capital Humano; Ciencia, Tecnología e Innovación; e Instituciones y Finanzas públicas.

sociedad y economía • e-ISSN: 2389-9050 • No. 39, 2020 • pp. 80-112 
departamental, utilizando la metodología subjetiva de ponderación, del Foro Económico Mundial, con 97variables agrupadas en 10 pilares 4 que a su vez están agrupados en tres factores 5 . Además, se han estimado otros indicadores de competitividad regional, como los del CID (2002), CRECE (2002), Espinosa y Albis (2005), y Lozano y Yoshida (2008), elaborados para un solo periodo.

Todos los indicadores de competitividad usan metodologías similares a las utilizadas por el Foro Económico Mundial (2014) y el Instituto Para la Gestión del Desarrollo (1996); los cuales, en su indicador, no tienen un marco teórico económico que identifique los fallos de mercado que conlleven a conflictos económicos entre las regiones e ignoran el enfoque que brinda la geografía económica para facilitar la comprensión de los fenómenos regionales (Lall, 2001; Lotero et al., 2009).

Los indicadores de competitividad regional de la CEPAL, CPC y Foro Económico Mundial (FEM) han recibido críticas sobre la cantidad de variables que utilizan para sus análisis. Esto conduce a problemas de excesiva prescripción de políticas y a la pérdida de coherencia entre el marco teórico y el conjunto de variables escogidas (Lall, 2001; Lotero et al., 2009). Algo que acarrea el uso de pilares o factores redundantes no acordes con la competitividad entre las regiones y que podrían omitirse utilizando la teoría de geografía económica (Lotero et al., 2009).

Además, los indicadores de competitividad de la CEPAL y del CPC de los departamentos colombianos no tienen cambios sustanciales en el periodo de estudio, por lo que las posiciones de los departamentos se mantienen igual. Es por ello que departamentos de la región Orinoquía/Amazonía, que tienen indicadores bajos, no alcanzarán a los departamentos que tienen

4 Instituciones; Infraestructura; Tamaño del Mercado; Educación Básica y Media; Salud; Sostenibilidad Ambiental; Educación Superior y Capacitación; Eficiencia de los Mercados; Sofisticación y Diversificación; e Innovación y Dinámica Empresarial.

5 Condiciones básicas, Eficiencia y Sofisticación e innovación. los primeros puestos, como Bogotá, Antioquia, Santander y Caldas, por contar (los últimos) con mayores indicadores (Ramírez y de Aguas, 2015).

En tal sentido, al evaluar las teorías que sustentan estos indicadores de competitividad se observa que solo miden a los departamentos de manera generalizada, con sus ventajas de aglomeración, y no estudian las características heterogéneas de cada región (Nava, Cernas y Becerril, 2017; Ramírez y de Aguas, 2015).

\subsection{Metodología de evaluación de indices de competitividad}

Se usa como proxy el PIB real per cápita (Sánchez, 201l; Krugman y Wells, 2006; Hall y Jones, 1999), con el fin de evaluar la relación entre los indicadores de competitividad de la CEPAL y del CPC con el nivel de productividad. Lo anterior para determinar si los índices de competitividad inciden en la productividad per cápita de los departamentos (Huber y Mungaray, 2017).

Para la evaluación se estima un modelo econométrico panel data, utilizando como variable dependiente el PIB real per cápita departamental y el indicador de competitividad de la CEPAL y del CPC como variable independiente.

Se estimaron dos modelos econométricos, el primero (ecuación 1), para la CEPAL, en el cual la variable dependiente es el PIB real per cápita en escala logarítmica y la variable independiente es el índice de competitividad departamental (ICD) elaborado por la CEPAL. El segundo modelo (ecuación 2), para el CPC, donde la variable dependiente es el PIB real per cápita en escala logarítmica y la variable independiente es el índice departamental de competitividad (IDC), elaborado por el CPC.

Para el modelo propuesto, se especifica como:

$$
Y_{i t}=v_{i}+\beta_{1} I C D_{i t}+e_{i t} \quad(1)
$$

Donde $Y_{i t}$ es el logaritmo del PIB real per cápita del departamento $i$ en el año $t ; v_{i}$ es un vector de variables dicotómicas para cada departamento; $\beta_{1}$ representa el coeficiente del valor del 
índice de competitividad departamental de la CEPAL $i$ en el periodo $t$; y $e_{i t}$ es el error aleatorio.

$$
Y_{i t}=v_{i}+\beta_{1} I D C_{i t}+e_{i t}(2)
$$

Donde $Y_{i t}$ es el logaritmo del PIB real per cápita del departamento $i$ en el año $t ; v_{i}$ es un vector de variables dicotómicas para cada departamento; $\beta$ representa el coeficiente del valor del índice departamental de competitividad del CPC $i$ en el periodo $t$; y $e_{i t}$ es el error aleatorio.

\subsection{Resultados de la evaluación de indices de competitividad CEPAL Y CPC}

El resultado del primer modelo (Tabla 1), muestra que el índice de competitividad de la CEPAL impacta positivamente el PIB real per cápita y significativo en un $99 \%$. En cambio, el segundo modelo del índice de competitividad departamental del Consejo privado también lo es, pero con nivel de significancia menor del $90 \%$.

Para el modelo de la CEPAL, 20 de los 32 departamentos resultaron significativos y positivos para la relación PIB per cápita y el índice de competitividad departamental, es decir, el $62 \%$. Los departamentos con mayores coeficientes son Casanare (1,6), Arauca $(1,2)$ y Meta $(1,1)$; es decir, cuando el índice de competitividad departamental de Casanare, Arauca y Meta, aumenta en una unidad, el PIB real per cápita o productividad de estos departamentos aumenta en $1,6 \%$, $1,2 \%$ y $1,1 \%$, respectivamente. Los departamentos con menores coeficientes son Valle del cauca $(0,28)$, Atlántico $(0,20)$ y Antioquia $(0,15)$, quienes tienen mayor participación en el PIB de Colombia y cuentan con aglomeraciones que originan marcadas diferencias de sus ventajas competitivas frente a los demás departamentos.

Por otro lado, los 11 departamentos que no resultaron significativos, es decir el $38 \%$, son Caldas, Caquetá, Cauca, Chocó, Guaviare, Magdalena, Nariño, Norte de Santander, Quindío, Risaralda y Sucre.

Para el Consejo Privado, 16 de los 26 departamentos resultaron significativos para la relación
PIB per cápita y el índice departamental de competitividad, es decir, el 61,5\%. Los departamentos con mayores coeficientes son Casanare $(0,90)$, Meta $(0,82)$ y Santander $(0,42)$; es decir, cuando el índice departamental de competitividad de Casanare, Meta y Santander, aumenta en una unidad, el PIB real per cápita o productividad de estos departamentos aumenta en 0,90\%, $0,82 \%$ y $0,42 \%$, respectivamente. Entre los departamentos que tienen coeficientes negativos se destacan Chocó $(-0,46)$, Norte de Santander $(-0,53)$, Quindío $(-0,57)$, Magdalena $(-0,59)$ y Sucre $(-0,61)$, caracterizados por su ruralidad y desaglomeración; hechos que no permiten aprovechar sus potenciales productivos de manera eficiente (Ramírez y de Aguas, 2015).

Por otro lado, los 10 departamentos (Antioquia, Bolívar, Cauca, Cesar, Cundinamarca, Huila, Guajira, Putumayo, Tolima y Valle del Cauca) que no resultaron significativos estadísticamente, suman el $38,4 \%$ de los departamentos. Es decir, el índice de competitividad departamental del CPC no incide en la productividad de tales departamentos. En conclusión, se puede observar que, para algunos departamentos, el indicador de competitividad no incide en su productividad.

\subsection{Metodología de evaluación de factores de competitividad de la CEPAL y del CPC}

Llevada a cabo con el fin de observar cuáles factores de los indicadores de competitividad de la CEPAL y el CPC están relacionados con el PIB real per cápita. Para ello, se estiman dos modelos panel data. El primero para la CEPAL (ecuación 3), la variable dependiente es el logaritmo del PIB real per cápita de cada departamento y las variables independientes son los cinco factores que integran el índice de competitividad: fortaleza económica (For); infraestructura (Inf); capital humano (CAP); ciencia, tecnología e innovación (CTI); e instituciones, gestión y finanza públicas (PUBL), para el periodo de 2000 - 2015. En el segundo modelo (ecuación 4), la variable dependiente es el logaritmo del PIB real per 
Tabla 1. Resultado modelos panel data PIB real per cápita departamental e índices de competitividad regional de la CEPAL y CPC

\begin{tabular}{|c|c|c|c|c|c|c|c|}
\hline \multicolumn{4}{|c|}{ Modelo CEPAL con tasa del PIB real per cápita } & \multicolumn{4}{|c|}{ Modelo CPC con tasa del PIB real per cápita } \\
\hline Departamento & Coeficientes & $\begin{array}{c}\text { Error } \\
\text { estándar }\end{array}$ & $p>[z]$ & Departamento & Coeficientes & $\begin{array}{c}\text { Error } \\
\text { estándar }\end{array}$ & $p>[z]$ \\
\hline ICD & 0,0186259 & 0,0015627 & 0,000 & IDC & 0,0064253 & 0,0037268 & 0,089 \\
\hline Cons & 12,23013 & 0,163054 & 0,000 & Cons & 13,84311 & 0,3059478 & 0,000 \\
\hline Casanare & 1,611763 & 0,1263397 & 0,000 & Casanare & 0,9009919 & 0,1455714 & 0,000 \\
\hline Arauca & 1,24082 & 0,1380725 & 0,000 & Meta & 0,8228912 & 0,1409802 & 0,000 \\
\hline Meta & 1,116497 & 0,1157623 & 0,000 & Santander & 0,4225752 & 0,0915464 & 0,000 \\
\hline Santander & 0,6830531 & 0,093492 & 0,000 & Boyacá & 0,3176053 & 0,1174897 & 0,008 \\
\hline Cesar & 0,6735652 & 0,1257929 & 0,000 & Córdoba & $-0,271203$ & 0,1514339 & 0,077 \\
\hline Putumayo & 0,6690314 & 0,1507824 & 0,000 & Caldas & $-0,3433514$ & 0,0818971 & 0,000 \\
\hline San Andrés & 0,6141795 & 0,121229 & 0,000 & Risaralda & $-0,351834$ & 0,1008296 & 0,001 \\
\hline Bolívar & 0,5778502 & 0,1184673 & 0,000 & Nariño & $-0,3646988$ & 0,1545825 & 0,021 \\
\hline Vichada & 0,5746019 & 0,1828676 & 0,002 & Caquetá & $-0,402487$ & 0,1885159 & 0,036 \\
\hline La Guajira & 0,5676204 & 0,1344409 & 0,000 & Atlántico & $-0,4415294$ & 0,1185474 & 0,000 \\
\hline Guainía & 0,5352343 & 0,201537 & 0,009 & Chocó & $-0,4637179$ & 0,225694 & 0,043 \\
\hline Boyacá & 0,49623 & 0,108606 & 0,000 & N. de Santander & $-0,5376881$ & 0,1494843 & 0,001 \\
\hline Vaupés & 0,4162991 & 0,2020999 & 0,042 & Quindío & $-0,5767427$ & 0,1296249 & 0,000 \\
\hline Huila & 0,3854837 & 0,1155438 & 0,001 & Magdalena & $-0,5997542$ & 0,1634304 & 0,000 \\
\hline Córdoba & 0,3343113 & 0,1327363 & 0,013 & Sucre & $-0,6100965$ & 0,1574222 & 0,000 \\
\hline Tolima & 0,3070994 & 0,1137872 & 0,008 & & & & \\
\hline Amazonas & 0,2913956 & 0,1476171 & 0,051 & & & & \\
\hline Valle & 0,2846501 & 0,0906989 & 0,002 & & & & \\
\hline Atlántico & 0,206385 & 0,1007831 & 0,043 & & & & \\
\hline Antioquia & 0,1518329 & 0,0859773 & 0,080 & & & & \\
\hline
\end{tabular}

Fuente: elaboración propia. 
cápita y las variables independientes son los tres factores que integran el índice de competitividad del CPC: condiciones básicas (CB), eficiencia (EFI) y sofisticación e innovación (SOF).

Para el modelo propuesto, se especifica como:

$$
Y_{i t}=v_{i}+\beta_{1} F O R_{i t}+\beta_{2} I N F_{i t}+\beta_{3} C A P_{i t}+\beta_{4} C T I_{i t}+\beta_{5} P U B L_{i t}+e_{i t} \text { (3) }
$$

Donde $v_{i}$ es un vector de variables dicotómicas para cada departamento, $\beta_{1 . n}=$ factor de competitividad departamental de la CEPAL del i-esimo departamentos $i$ en el periodo $t$; y $e_{i t}$ es el error aleatorio.

$$
Y_{i t}=v_{i}+\beta_{1} C B_{i t}+\beta_{2} \mathrm{EFI}_{i t}+\beta_{3} \mathrm{SOF}_{i t}+e_{i t}(4)
$$

Donde $v_{i}$ es un vector de variables dicotómicas para cada departamento, $\beta_{1 . n}=$ factor de competitividad departamental del CPC i-esimo departamentos $i$ en el periodo $t$; $y e_{i t}$ es el error aleatorio.

\subsection{Resultados de la evaluación de los factores de competitividad de la CEPAL Y CPC}

El tercer modelo relaciona el PIB real per cápita con los factores de competitividad departamental de la CEPAL (Tabla 2) en este se muestra que cuatro de los cinco factores resultaron positivos y significativos; los factores fortaleza económica $(0,009)$, capital humano $(0,003)$, ciencia y tecnología e innovación $(0,002)$ e instituciones, gestión y finanza $(0,001)$. El factor que no resultó significativo con el PIB per cápita fue infraestructura.

En la relación entre el PIB real per cápita y los factores de competitividad departamental de la CPC, solo uno de los tres factores de competitividad resultó significativo con un nivel de significancia del 99\%; es el factor sofisticación e innovación $(0,006)$, que estudia la capacidad que tienen los departamentos para producir bienes y servicios de alto valor agregado, así como la fortaleza de su tejido empresarial y la ciencia, tecnología e innovación (CPC, 2013).

Se concluye que el indicador de competitividad de la CEPAL es el que con sus factores está relacionado con la productividad de los departamentos, no se puede decir lo mismo con el indicador del CPC.

\section{Propuesta de Indicador de competitividad regional departamental}

La propuesta de este trabajo es crear un indicador de competitividad para los departamentos de Colombia basado en las teorías de aglomeraciones, economía geográfica, economía institucional y capital humano. Así mismo, considerando las críticas a los indicadores regionales de competitividad hechas por Lotero et al. (2009), Krugman (1995) y Lall (2001), en lo referente al gran número de variables en los indicadores y la coherencia entre la solidez metodológica y el marco teórico y conceptual. Además, el trabajo se propone analizar la correspondencia de los indicadores regionales con la productividad de los departamentos.

\subsection{Metodología econométrica componentes principales.}

Se utilizó un modelo factorial de análisis de componentes principales (ACP), método multivariante cuyo objetivo es convertir un problema de información estadística muy compleja (muchas variables de tipo cuantitativo medidas en cada unidad de observación) en otro casi equivalente, pero más manejable (pocas variables), sin pérdida significativa de información. La técnica obtiene combinaciones lineales de todas las variables originales que sean ortogonales entre sí. De esta forma se obtiene un subconjunto de nuevas variables que son independientes entre sí, pero que están altamente correlacionadas con las variables originales, denominadas componentes principales, que ordenadas en forma decreciente según su varianza, permiten explicar el porcentaje de variabilidad de los datos por cada componente. Los valores y vectores característicos se obtienen diagonalizando la matriz de varianza y covarianza o la matriz de 
Tabla 2. Modelos panel data PIB real per cápita departamental y factores de competitividad de la CEPAL y el CPC

\begin{tabular}{|c|c|c|c|c|c|c|c|}
\hline \multicolumn{4}{|c|}{ Modelo CEPAL con tasa PIB real per cápita } & \multicolumn{4}{|c|}{ Modelo CPC con tasa PIB real per cápita } \\
\hline & Coeficientes & $\begin{array}{c}\text { Error } \\
\text { estándar }\end{array}$ & $p>[z]$ & Departamentos & Coeficientes & $\begin{array}{l}\text { Error } \\
\text { estándar }\end{array}$ & $\mathbf{p}>[\mathbf{z}]$ \\
\hline $\begin{array}{l}\text { Fortaleza } \\
\text { Económica }\end{array}$ & 0,0090367 & 0,0013576 & 0,000 & $\begin{array}{l}\text { Sofisticación e } \\
\text { Innovación }\end{array}$ & 0,0065742 & 0,0012521 & 0,000 \\
\hline Capital Humano & 0,0031296 & 0,0008766 & 0,001 & Cons & 13,72465 & 0,1241683 & 0,000 \\
\hline $\begin{array}{l}\text { Ciencia, Tecnología } \\
\text { e Innovación }\end{array}$ & 0,0028133 & 0,0015123 & 0,065 & Casanare & 1,146911 & 0,0995902 & 0,000 \\
\hline $\begin{array}{c}\text { Instituciones, } \\
\text { Gestión y Finanzas } \\
\text { Publicas }\end{array}$ & 0,0018604 & 0,0008855 & 0,038 & Meta & 1,074243 & 0,1009122 & 0,000 \\
\hline Cons & 12,41987 & 0,1864393 & 0,000 & Santander & 0,5759959 & 0,0611181 & 0,000 \\
\hline Casanare & 1,504911 & 0,1537954 & 0,000 & Boyacá & 0,5297658 & 0,0806822 & 0,000 \\
\hline Arauca & 1,139881 & 0,1578244 & 0,000 & Putumayo & 0,4060847 & 0,1183887 & 0,001 \\
\hline Meta & 1,029963 & 0,1490739 & 0,000 & Cesar & 0,2319711 & 0,1003 & 0,021 \\
\hline Santander & 0,7013211 & 0,1132084 & 0,000 & Cauca & 0,2242339 & 0,0694003 & 0,001 \\
\hline Cesar & 0,6364824 & 0,1577258 & 0,000 & Huila & 0,1546071 & 0,0930151 & 0,096 \\
\hline La Guajira & 0,5242391 & 0,1677556 & 0,002 & Cundinamarca & 0,1299551 & 0,051029 & 0,011 \\
\hline Boyacá & 0,4707566 & 0,1321705 & 0,001 & Risaralda & $-0,1754919$ & 0,0696849 & 0,012 \\
\hline Putumayo & 0,4700232 & 0,1695937 & 0,006 & Nariño & $-0,1757754$ & 0,0884369 & 0,047 \\
\hline San Andrés & 0,4523535 & 0,1497692 & 0,003 & Caldas & $-0,1982824$ & 0,0581963 & 0,001 \\
\hline Bolívar & 0,4441209 & 0,1446523 & 0.003 & Chocó & $-0,2997079$ & 0,1066641 & 0,005 \\
\hline Huila & 0,3887993 & 0,1510983 & 0,011 & Quindío & $-0,3390673$ & 0,0894285 & 0,000 \\
\hline Valle & 0,09249 & 0,1137214 & 0,008 & Atlántico & $-0,3527481$ & 0,0619412 & 0,000 \\
\hline \multirow[t]{3}{*}{ Tolima } & 0,303272 & 0,1469371 & 0,041 & N. de Santander & $-0,3686143$ & 0,0831479 & 0,000 \\
\hline & & & & Sucre & $-0,4036407$ & 0,0925576 & 0,000 \\
\hline & & & & Magdalena & $-0,4441702$ & 0,0847217 & 0,000 \\
\hline
\end{tabular}

Fuente: elaboraci propios. 
correlaciones (Villardón, 2002; Salinas, Albornoz, Reyes, Erazo e Ide, 2006).

Consecutivamente, cada departamento obtiene una puntuación en cada uno de los componentes principales seleccionados, ordenando las observaciones con base en la información multivariante. Además, se transforman mediante la técnica de normalización mínimo-máximo (ecuacion 5), como factor de escala, cambiando las puntuaciones en escalas de $\mathrm{O}$ a 100, donde $\mathrm{O}$ es el de menos puntaje y 100 el mayor (Jain, Nandakumar y Ross, 2005).

$$
y_{c i}=\frac{\left(\text { valor }_{c i}-\min _{i}\right)}{\left(\max _{i}-\min _{i}\right)} * 100
$$

Donde $y_{c i}$ es el puntaje normalizado del departamento $c$ en el indicador $i$; el valor ${ }_{c i}$ es el valor real del departamento c en el indicador $i$; $\min _{i}$ es el valor mínimo en el indicador $i$, y $\max _{i}$ es el valor máximo en el indicador $i$. Adicionalmente, para el análisis de los resultados, el indicador se divide en tres niveles: alto de 100 a 70; medio de 69 a 30; $y$ bajo de 29 a 0.

\subsection{Base de datos}

Se estimaron siete índices de competitividad regional departamental: el de aglomeración, fuerzas centrifugas, geografía económica, institucional, capital humano, ciencia, tecnología e innovación e infraestructura. Se utilizaron 26 variables, obtenidas de datos secundarios de diferentes fuentes, para el periodo 2013-2016. Además, fueron escogidas teniendo en cuenta criterios teóricos, empíricos y estadísticos: uno, las variables deben estar relacionadas con las teorías de aglomeraciones, capital humano, economía geográfica y economía institucional. Dos, las variables escogidas deben tener un sustento empírico en trabajos similares o relacionados a nivel nacional e internacional. Tres, las variables no deben ser escogidas a priori, sino por medio de su significancia o correlación con respeto al PIB per cápita. Y cuatro, las variables deben estar disponibles y actualizadas. Por último, el indicador propuesto presenta una ventaja en términos de reducción del $63 \%$ de variables con respeto al indicador de la CEPAL, y con el CPC una reducción del 73\% (Tabla 3).

\subsection{Resultados de índices regionales de competitividad departamental de Colombia 2013-2014-2015-2016}

El indicador propuesto en el trabajo presenta siete índices determinantes para el Indicador Regional de Competitividad Departamental en Colombia (IRCD), estos son: aglomeración, fuerzas centrifugas, infraestructura, economía geográfica, institucional, capital humano y ciencia y tecnología; los cuales son coherentes con la competitividad regional que se enmarca en las teorías de aglomeraciones, economía geográfica, economía institucional y capital humano.

Tabla 3. Operacionalización de variables del índice de competitividad regional departamental

\begin{tabular}{lccc}
\hline Factor & Variable & Unidad & Fuente \\
\hline \multirow{2}{*}{$\begin{array}{c}\text { Factor } \\
\text { Aglomerticipación PIB }\end{array}$} & Porcentaje \\
& $\begin{array}{c}\text { Participación PIB industrial } \\
\text { departamental en el PIB } \\
\text { industrial nacional }\end{array}$ & Porcentaje & DANE \\
& $\begin{array}{c}\text { Participación PIB de Servicios } \\
\text { departamental en el PIB de } \\
\text { servicios nacional } \\
\end{array}$ & Población ocupada & DANE \\
& & Miles de personas & DANE \\
\hline
\end{tabular}


Tabla 3. Operacionalización de variables del índice de competitividad regional departamental

\begin{tabular}{|c|c|c|c|}
\hline \multirow{3}{*}{$\begin{array}{c}\text { Factor Fuerzas } \\
\text { Centrifugas }\end{array}$} & PIB agrícola & Miles de millones & DANE \\
\hline & PIB minero & Miles de millones & DANE \\
\hline & $\begin{array}{l}\text { Irradiación global horizontal } \\
\text { media diaria }\end{array}$ & Kwh/M2 & $\begin{array}{c}\text { Instituto de Hidrología, } \\
\text { Meteorología y Estudios } \\
\text { Ambientales-Ideam }\end{array}$ \\
\hline \multirow{4}{*}{$\begin{array}{l}\text { Factor } \\
\text { Economía } \\
\text { Geográfica }\end{array}$} & $\begin{array}{l}\text { Costo total de transporte } \\
\text { terrestre a puertos por } \\
\text { departamento }\end{array}$ & Dólares por tonelada & $\begin{array}{c}\text { Sicetac - Ministerio de } \\
\text { Transporte }\end{array}$ \\
\hline & $\begin{array}{c}\text { Costo de transporte por } \\
\text { departamento a las ciudades } \\
\text { capitales }\end{array}$ & Dólares por tonelada & $\begin{array}{c}\text { Sicetac - Ministerio de } \\
\text { Transporte }\end{array}$ \\
\hline & Toneladas de residuos & Porcentaje & $\begin{array}{l}\text { Superintendencia de } \\
\text { Servicios Públicos } \\
\text { Domiciliarios }\end{array}$ \\
\hline & Tasa afectación desastres & $\begin{array}{c}\text { Población afectada por } \\
\text { desastres naturales por cada } \\
100.000 \text { habitantes }\end{array}$ & $\begin{array}{c}\text { Instituto de Hidrología, } \\
\text { Meteorología Y Estudios } \\
\text { Ambientales-Ideam }\end{array}$ \\
\hline \multirow{3}{*}{$\begin{array}{l}\text { Factor } \\
\text { Economía } \\
\text { Institucional }\end{array}$} & Índice de desempeño integral & $0-98$ & $\begin{array}{c}\text { Departamento Nacional } \\
\text { de Planeación }\end{array}$ \\
\hline & Índice de desempeño fiscal & $0-99$ & $\begin{array}{c}\text { Departamento Nacional } \\
\text { de Planeación }\end{array}$ \\
\hline & Índice de gobierno abierto & $0-100$ & $\begin{array}{c}\text { Procuraduría General de } \\
\text { la Nación }\end{array}$ \\
\hline \multirow{4}{*}{$\begin{array}{c}\text { Factor Capital } \\
\text { Humano }\end{array}$} & $\begin{array}{l}\text { Demanda laboral de recién } \\
\text { graduados universitarios }\end{array}$ & $\begin{array}{c}\text { Cantidad de graduados } \\
\text { que cotizan en el mercado } \\
\text { laboral formal }\end{array}$ & $\begin{array}{c}\text { Observatorio Laboral de } \\
\text { Educación }\end{array}$ \\
\hline & $\begin{array}{l}\text { Cobertura neta en educación } \\
\text { secundaria }\end{array}$ & Porcentaje & Ministerio de Educación \\
\hline & $\begin{array}{c}\text { Cobertura neta en educación } \\
\text { media }\end{array}$ & Porcentaje & Ministerio de Educación \\
\hline & $\begin{array}{l}\text { Puntaje pruebas Saber } 11 \\
\text { (lenguaje, matemáticas y } \\
\text { ciencias) }\end{array}$ & $0-100$ & Icfes \\
\hline \multirow{4}{*}{$\begin{array}{l}\text { Factor Ciencia, } \\
\text { Tecnología e } \\
\text { Innovación }\end{array}$} & Solicitudes de patentes & $\begin{array}{c}\text { Número de solicitudes } \\
\text { de patentes de invención } \\
\text { presentadas por residentes y } \\
\text { por departamento }\end{array}$ & $\begin{array}{c}\text { Boletín estadístico } \mathrm{N}^{\circ} 7 \\
2017 \text {-Colciencias }\end{array}$ \\
\hline & Becas de maestría asignadas & $\begin{array}{l}\text { Número de becas de maestría } \\
\text { asignadas por departamento }\end{array}$ & $\begin{array}{c}\text { Boletín estadístico } \mathrm{N}^{\circ} 7 \\
2017 \text {-Colciencias }\end{array}$ \\
\hline & Becas de doctorado & $\begin{array}{l}\text { Número becas de doctorado } \\
\text { asignadas por departamento }\end{array}$ & $\begin{array}{c}\text { Boletín estadístico } \mathrm{N}^{\circ} 7 \\
2017 \text {-Colciencias }\end{array}$ \\
\hline & $\begin{array}{l}\text { Inversión en proyecto de } \\
\text { investigación }\end{array}$ & Millones & $\begin{array}{c}\text { Boletín estadístico } \mathrm{N}^{\circ} 7 \\
2017 \text {-Colciencias }\end{array}$ \\
\hline
\end{tabular}


Tabla 3. Operacionalización de variables del índice de competitividad regional departamental

\begin{tabular}{|c|c|c|c|}
\hline \multirow{4}{*}{$\begin{array}{c}\text { Factor } \\
\text { Infraestructura }\end{array}$} & Densidad de vías secundarias & $\begin{array}{l}\text { Kilómetros de vías } \\
\text { secundarias pavimentadas } \\
\text { por cada } 100 \text { Kilómetros de } \\
\text { superficie }\end{array}$ & Invías-Dane \\
\hline & Inversión en comunicaciones & Millones & $\begin{array}{c}\text { Departamento Nacional } \\
\text { de Planeación }\end{array}$ \\
\hline & Inversión en minas y energía & Millones & $\begin{array}{c}\text { Departamento Nacional } \\
\text { de Planeación }\end{array}$ \\
\hline & Inversión en transporte & Millones & $\begin{array}{c}\text { Departamento Nacional } \\
\text { de Planeación }\end{array}$ \\
\hline
\end{tabular}

Fuente: elaboración propia.

IRCD = $f$ (Aglomeración, Fuerzas centrífugas, Infraestructura, Ciencia y Tecnología, Economía geográfica, Economía institucional y Capital humano)

\section{4·3.1 Índice de aglomeración}

El índice de aglomeración está constituido por las variables de participación del PIB departamental en el nacional, participación de la industria departamental en la nacional, participación de los servicios departamentales en los servicios nacionales y población ocupada laboralmente. Las variables fueron elegidas teniendo en cuenta la teoría de aglomeración y el nivel de significancia con el PIB real per cápita departamental. Según el modelo de componentes principales la variable que presenta mayor representatividad en el índice es participación del PIB departamental con 0,997 de carga factorial.

Aglomeración $=f$ (Participación del PIB departamental en el nacional; Participación de la industria departamental en la nacional; Participación de los servicios departamentales en los servicios nacionales; Población ocupada) (7)

Los resultados del índice de aglomeración propuesto en este trabajo (Tabla 4), muestran que el departamento con mayor indicador de competitividad, y el único en el nivel alto en el periodo de estudio y con gran diferencia de los demás, es Cundinamarca/Bogotá. Esto gracias a su población de más de ocho millones de habitantes, que incentiva la aglomeración de industrias y consumidores, lo que es una ventaja competitiva frente a otros departamentos. Además, se observa que la aglomeración de la actividad económica produce grandes diferencias en el indicador entre los departamentos.

En el nivel medio se encuentran los departamentos de Antioquia y Valle del Cauca, igualmente debido a sus poblaciones que sobrepasan los dos millones de habitantes y sus significativos aportes en el PIB nacional, según datos del DANE. Tales son razones de peso para que las empresas destinen su ubicación y producción hacia estos mercados domésticos, razones reforzadas por factores geográficos asociados con la aglomeración. Factores tales como la proximidad geográfica, las externalidades, los encadenamientos con la demanda y la fragmentación territorial del país por la persistencia de los altos costos de transporte (Lotero, 2007).

En el nivel bajo se encuentran 29 departamentos, es decir, el $90 \%$ está en el nivel bajo. Por otro lado, cabe destacar el hecho de que Bolívar esté por encima del Atlántico, gracias a la ventaja comparativa de Cartagena por la ubicación de su refinería de petróleo y gran puerto (Acosta, 2012). 


\begin{tabular}{|c|c|c|c|c|c|c|c|c|}
\hline Dep. & $\begin{array}{c}\text { Posición } \\
2013\end{array}$ & $\begin{array}{c}\text { Índice de } \\
\text { Aglomeración } \\
\text { Año } 2013\end{array}$ & $\begin{array}{c}\text { Posición } \\
2014\end{array}$ & $\begin{array}{c}\text { Índice de } \\
\text { Aglomeración } \\
\text { Año } 2014\end{array}$ & $\begin{array}{c}\text { Posición } \\
2015\end{array}$ & $\begin{array}{c}\text { Índice de } \\
\text { Aglomeración } \\
\text { Año } 2015\end{array}$ & $\begin{array}{c}\text { Posición } \\
2016\end{array}$ & $\begin{array}{l}\text { Índice de } \\
\text { Aglomeración } \\
\text { Año } 2016\end{array}$ \\
\hline Cund/Bogotá & 1 & 100,0 & 1 & 100,0 & 1 & 100,0 & 1 & 100,0 \\
\hline Antioquia & 2 & 47,3 & 2 & 47,8 & 2 & 48,0 & 2 & 48,5 \\
\hline Valle & 3 & 35,2 & 3 & 35,3 & 3 & 36,2 & 3 & 36,5 \\
\hline Santander & 4 & 27,9 & 4 & 30,8 & 4 & 29,2 & 4 & 27,5 \\
\hline Bolívar & 5 & 17,9 & 5 & 16,7 & 6 & 14,7 & 5 & 17,5 \\
\hline Atlántico & 6 & 15,2 & 6 & 15,5 & 5 & 16,0 & 6 & 16,6 \\
\hline Boyacá & 7 & 9,1 & 7 & 9,4 & 7 & 9,8 & 7 & 9,7 \\
\hline Tolima & 9 & 7,9 & 9 & 7,9 & 8 & 8,0 & 8 & 8,1 \\
\hline Cauca & 11 & 6,7 & 11 & 6,8 & 9 & 7,3 & 9 & 7,5 \\
\hline Nariño & 10 & 6,8 & 10 & 6,9 & 11 & 6,9 & 10 & 7,1 \\
\hline Córdoba & 12 & 6,5 & 12 & 6,6 & 12 & 6,6 & 11 & 6,5 \\
\hline Meta & 8 & 9,0 & 8 & 8,2 & 10 & 7,1 & 12 & 6,3 \\
\hline $\begin{array}{c}\text { Norte de } \\
\text { Santander }\end{array}$ & 13 & 6,1 & 13 & 6,1 & 13 & 6,0 & 13 & 6,1 \\
\hline Risaralda & 15 & 5,4 & 15 & 5,5 & 15 & 5,6 & 14 & 5,8 \\
\hline Huila & 14 & 5,8 & 14 & 5,8 & 14 & 5,8 & 15 & 5,8 \\
\hline Caldas & 16 & 5,3 & 16 & 5,4 & 16 & 5,6 & 16 & 5,6 \\
\hline Magdalena & 17 & 4,9 & 17 & 4,8 & 17 & 4,8 & 17 & 4,8 \\
\hline Cesar & 18 & 4,7 & 18 & 4,7 & 18 & 4,7 & 18 & 4,7 \\
\hline Sucre & 20 & 3,3 & 20 & 3,4 & 19 & 3,4 & 19 & 3,4 \\
\hline La Guajira & 19 & 3,4 & 19 & 3,4 & 20 & 3,3 & 20 & 3,4 \\
\hline Quindío & 22 & 2,7 & 22 & 2,7 & 21 & 2,7 & 21 & 2,8 \\
\hline Casanare & 21 & 2,8 & 21 & 2,7 & 22 & 2,4 & 22 & 2,0 \\
\hline Caquetá & 23 & 1,6 & 23 & 1,7 & 23 & 1,6 & 23 & 1,8 \\
\hline Chocó & 24 & 1,4 & 24 & 1,2 & 24 & 1,3 & 24 & 1,4 \\
\hline Arauca & 25 & 1,0 & 25 & 0,9 & 25 & 0,8 & 25 & 0,8 \\
\hline Putumayo & 26 & 0,8 & 26 & 0,7 & 26 & 0,6 & 26 & 0,5 \\
\hline San Andrés & 27 & 0,4 & 27 & 0,4 & 27 & 0,3 & 27 & 0,3 \\
\hline Guaviare & 28 & 0,2 & 28 & 0,2 & 28 & 0,2 & 28 & 0,2 \\
\hline Amazonas & 29 & 0,1 & 29 & 0,1 & 29 & 0,1 & 29 & 0,1 \\
\hline Vichada & 30 & 0,1 & 30 & 0,1 & 30 & 0,1 & 30 & 0,0 \\
\hline Guainía & 31 & 0,0 & 31 & 0,0 & 31 & 0,0 & 31 & 0,0 \\
\hline Vaupés & 32 & 0,0 & 32 & 0,0 & 32 & 0,0 & 32 & 0,0 \\
\hline
\end{tabular}

Fuente: elaboración propia. 
Los departamentos con puntajes más bajos son Arauca, Putumayo, San Andrés, Guaviare, Amazonas, Vichada, Guainía y Vaupés, territorios caracterizados por su baja población, casi nula producción industrial y su baja densidad poblacional, factores que van en contravía con la teoría de aglomeración. Es importante indicar que los puestos de los departamentos, en los tres años analizados, no cambian en el sentido de que difícilmente cambie la estructura productiva de los mismos.

\subsection{2 Índice fuerzas centrífugas}

El índice de fuerzas centrífugas está constituido por las variables del PIB minero departamental, PIB agrícola departamental y la irradiación global de energía solar que ostente el departamento; elegidas teniendo en cuenta la teoría de aglomeraciones con fuerzas centrifugas y su nivel de significancia con el PIB real per cápita departamental. Según el modelo de compontes principales, la variable que presenta mayor carga factorial en el índice es el PIB minero con 0,754 .

\section{Fuerzas centrífugas $=f(P I B$ minero departamental, PIB agrícola departamental y Energía Solar) (8)}

Entre los resultados del índice de fuerzas centrífugas propuesto en el trabajo (Tabla 5), se encuentra que los departamentos líderes en todo el periodo de estudio y que están en el nivel alto son Meta y Antioquia, por el hecho de ser los mayores productores en Colombia de mineria y agricultura, respectivamente. Sin embargo, en el 2016, el departamento del Cesar entró en el nivel alto gracias, según datos del DANE, a un aumento de la producción minera.

En el nivel medio se encuentran, en promedio, el $40 \%$ de los departamentos del país y se destacan los de Boyacá y Cundinamarca, que en los primeros años están entre los cuatro primeros, no obstante, son desplazados por el Cesar y la Guajira en los últimos años, según datos del DANE, los cuales han tenido un aumento de la producción minera y también por el gran potencial de irradiación solar que presenta la región Caribe (UPME -Unidad de Planeación Minero Energética-, 2005; Chamorro, Ortiz y Viana, 2015).

En el nivel bajo se encuentran, en promedio, el $53 \%$ de los departamentos del país. Además, los que tienen menor puntaje son Vaupés, Amazonas, Guaviare, Caquetá, Chocó y Putumayo, que resaltan por ser regiones geográficamente aisladas, con falta de conectividad vial, con condiciones climáticas extremas y estructuras económicas en sectores con poco valor agregado, condiciones que dificultan el crecimiento económico (Meisel, Bonilla y Sánchez, 2013; Bonet, 2007). Es importante resaltar que el índice de competitividad para los departamentos ha cambiado en el tiempo para algunos de estos; algo que se debe al incentivo, en las políticas del Gobierno, a la explotación minera.

\subsection{3 Índice de infraestructura}

El índice de infraestructura está constituido por las variables de inversión en transporte, inversión en comunicación, inversión en minas y energía y densidad en vías secundarias. Variables elegidas teniendo en cuenta la teoría de aglomeración y su nivel de significancia con el PIB real per cápita departamental. Según el modelo de componentes principales la variable que presenta mayor carga factorial es inversión en minas y energía con 0,908 .

\section{Infraestructura $=f$ (Inversión en transporte; Inversión en comunicación; Inversión en minas y energía y Densidad de vías secundarias) (9)}

Los resultados del índice de infraestructura propuesto en el trabajo (Tabla 6), presentan a Cundinamarca/Bogotá en el primer lugar, debido a que Bogotá es el centro urbano más grande de Colombia. Éste desarrolla un círculo virtuoso gracias a la urbanización y a las inversiones en transporte, comunicaciones, servicios públicos y vías secundarias, que son las mayores del país, para poder abastecer la demanda de servicios 
Estimación y comparación de la competitividad regional en Colombia

Tabla 5. Índice fuerza centrífuga, 2013-2016

\begin{tabular}{|c|c|c|c|c|c|c|c|c|}
\hline Dep. & $\begin{array}{c}\text { Posición } \\
2013\end{array}$ & $\begin{array}{c}\text { Índice de } \\
\text { Fuerzas } \\
\text { Centrifugas } \\
\text { Año } 2013 \\
\end{array}$ & $\begin{array}{c}\text { Posición } \\
2014\end{array}$ & $\begin{array}{c}\text { Índice de } \\
\text { Fuerzas } \\
\text { Centrifugas } \\
\text { Año } 2014 \\
\end{array}$ & $\begin{array}{c}\text { Posición } \\
2015\end{array}$ & $\begin{array}{c}\text { Índice de } \\
\text { Fuerzas } \\
\text { Centrifugas } \\
\text { Año } 2015 \\
\end{array}$ & $\begin{array}{l}\text { Posición } \\
2016\end{array}$ & $\begin{array}{c}\text { Índice de } \\
\text { Fuerzas } \\
\text { Centrifugas } \\
\text { Año } 2016 \\
\end{array}$ \\
\hline Meta & 1 & 100,0 & 1 & 100,0 & 1 & 100,0 & 1 & 100,0 \\
\hline Antioquia & 2 & 70,4 & 2 & 73,2 & 2 & 74,7 & 2 & 76,8 \\
\hline Cesar & 7 & 45,2 & 5 & 51,2 & 4 & 55,9 & 3 & 73,1 \\
\hline La Guajira & 8 & 43,5 & 7 & 48,7 & 5 & 53,9 & 4 & 65,9 \\
\hline Boyacá & 3 & 52,6 & 3 & 58,0 & 3 & 56,9 & 5 & 63,6 \\
\hline $\begin{array}{l}\text { Cund/ } \\
\text { Bogotá }\end{array}$ & 4 & 51,5 & 4 & 55,2 & 6 & 53,6 & 6 & 55,3 \\
\hline Casanare & 5 & 45,6 & 8 & 48,6 & 8 & 48,2 & 7 & 51,4 \\
\hline Tolima & 6 & 45,5 & 6 & 49,8 & 7 & 49,9 & 8 & 50,3 \\
\hline Valle & 9 & 43,5 & 9 & 47,9 & 9 & 45,4 & 9 & 46,3 \\
\hline Santander & 10 & 36,7 & 10 & 41,2 & 10 & 39,8 & 10 & 40,5 \\
\hline Magdalena & 11 & 34,6 & 11 & 38,6 & 11 & 38,3 & 11 & 39,8 \\
\hline Bolívar & 14 & 33,0 & 13 & 35,9 & 12 & 36,7 & 12 & 38,5 \\
\hline Arauca & 12 & 33,5 & 14 & 35,3 & 14 & 35,9 & 13 & 37,9 \\
\hline Huila & 13 & 33,2 & 12 & 37,5 & 13 & 36,5 & 14 & 36,7 \\
\hline Atlántico & 15 & 27,5 & 15 & 31,3 & 15 & 31,5 & 15 & 34,2 \\
\hline Córdoba & 16 & 26,8 & 16 & 29,5 & 16 & 29,9 & 16 & 32,9 \\
\hline San Andrés & 17 & 22,7 & 17 & 26,1 & 17 & 26,9 & 17 & 29,5 \\
\hline Vichada & 22 & 18,4 & 21 & 21,0 & 20 & 21,7 & 18 & 23,8 \\
\hline Sucre & 18 & 20,0 & 19 & 21,9 & 19 & 22,0 & 19 & 23,4 \\
\hline Cauca & 19 & 19,6 & 20 & 21,8 & 18 & 22,1 & 20 & 22,7 \\
\hline Caldas & 20 & 19,5 & 18 & 21,9 & 21 & 21,4 & 21 & 22,0 \\
\hline Nariño & 21 & 19,2 & 22 & 20,2 & 22 & 20,5 & 22 & 21,8 \\
\hline $\begin{array}{c}\text { Norte de } \\
\text { Santander }\end{array}$ & 23 & 16,4 & 23 & 17,8 & 23 & 17,3 & 23 & 18,4 \\
\hline Quindío & 24 & 16,2 & 24 & 17,7 & 24 & 17,2 & 24 & 17,9 \\
\hline Guainía & 26 & 13,7 & 26 & 15,7 & 25 & 16,2 & 25 & 17,7 \\
\hline Risaralda & 25 & 15,0 & 25 & 16,4 & 26 & 15,7 & 26 & 16,3 \\
\hline Caquetá & 27 & 10,6 & 27 & 11,9 & 27 & 11,8 & 27 & 12,5 \\
\hline Guaviare & 28 & 4,8 & 28 & 5,5 & 28 & 5,7 & 28 & 6,3 \\
\hline Chocó & 30 & 0,7 & 31 & 0,1 & 30 & 1,1 & 29 & 2,4 \\
\hline Putumayo & 29 & 2,7 & 29 & 2,7 & 29 & 1,3 & 30 & 0,5 \\
\hline Amazonas & 31 & 0,4 & 30 & 0,5 & 31 & 0,5 & 31 & 0,5 \\
\hline Vaupés & 32 & 0,0 & 32 & 0,0 & 32 & 0,0 & 32 & 0,0 \\
\hline
\end{tabular}

Fuente: elaboración propia. 
públicos y de vías que conecten a los agentes económicos. En el nivel alto solo aparece Antioquia, en el 2013, en los demás años no hay departamentos en tal nivel.

En el nivel medio se encuentran, en promedio, el 31\% de los departamentos de Colombia. Sin embargo, en el 2015 solo el $18 \%$ de los departamentos están en nivel medio. Además, se destacan Valle y Antioquía, es decir, son los departamentos con mayor participación en la producción nacional y tienen los principales mercados y áreas urbanas en Colombia, lo que requiere de una conexión vial y de unos servicios públicos que faciliten la actividad económica (Galvis, 2014). Por otro lado, se destaca el comportamiento de Nariño en los primeros puestos del indicador, debido a que se encuentra entre los departamentos con mayor inversión en transporte, minas y energías, la cual es necesaria por su ventaja geográfica, que permite movilización de mercancía a mercados locales e internacionales como Ecuador (Viloria, 2007; Pérez, 2005).

En el nivel bajo se encuentra el $64 \%$ de los departamentos. Sin embargo, se destaca que el porcentaje de departamentos en el nivel bajo aumenta al $80 \%$ en el 2015 y disminuye al $70 \%$ en el 2016. Por otro lado, los departamentos con los menores puntajes en niveles bajos son Guaviare, Vaupés, Guainía, Amazonas y Vichada.

Adicionalmente, se observa una relación entre los factores aglomeración e infraestructura, en el sentido de que la aglomeración de la población y la industria hace necesaria la inversión en infraestructura vial y comunicación. Sin embargo, algunos departamentos como Santander tienen un puesto menor en tal factor, lo mismo que Atlántico. Lo anterior implica que la inversión en infraestructura no solo depende de la aglomeración, si no de la voluntad política del ente territorial.

\subsection{4 Índice economía geográfica}

El índice economía geográfica está constituido por las variables de costo de transporte terrestre a puertos, costo de transporte terrestre a mercado interno, distancia a puertos marítimos, superficie cubierta por bosque, áreas protegidas; las variables se eligieron teniendo en cuenta la teoría de economía geográfica y sus relaciones con el PIB real per cápita. Según el análisis de compontes principales la variable que presenta mayor carga factorial es distancia a puertos marítimos con 0,902 .

Índice economía geográfica $=f($ Costo de transporte terrestre a puertos; Costo de transporte terrestre a mercado interno; Distancia a puertos marítimos; Superficie cubierta por bosque; Áreas

$$
\text { protegidas) (10) }
$$

Los resultados del índice de economía geográfica propuesto en el trabajo (Tabla 7), muestra que los departamentos en los primeros puestos pertenecen a la región Caribe, gracias a que tales departamentos tienen puertos marítimos. El Atlántico es líder del indicador al poseer puerto marítimo, tener bajos costos de transporte a puerto marítimo y poca superficie cubierta de bosque. Sin embargo, en el 2015, el Atlántico pierde su primera posición al aumentar los costos a puertos en $33 \%$ y al disminuir los costos a mercado interno en Sucre en $71 \%$. Por otro lado, en el nivel alto se encuentran, en promedio, el $47 \%$ de los departamentos del país. No obstante, en el 2016, el 71\% de los departamentos estaba en el nivel alto por disminución de los costos terrestres a mercados internos en 26 departamentos del país.

En el nivel medio se encuentran, en promedio, el $29 \%$ de los departamentos del país y se destacan Putumayo, Caquetá, Vichada, Chocó, Huila, Meta, Boyacá y Arauca. En el nivel bajo se encuentra el $13 \%$ de los departamentos, pertenecientes a la región de la Amazonía, caracterizados por su alto costo de transporte terrestre a puertos marítimos, a mercados y por sus áreas cubiertas de bosques. Estos son Vaupés, Amazonas, Guainía y Guaviare.

\subsection{5 Índice economía institucional}

El índice de economía institucional está constituido por las variables de índice de 
Tabla 6. Índice de infraestructura, 2013-2016

\begin{tabular}{|c|c|c|c|c|c|c|c|c|}
\hline Dep. & $\begin{array}{c}\text { Posición } \\
2013\end{array}$ & $\begin{array}{c}\text { Índice de } \\
\text { Infraestructura } \\
\text { Año } 2013 \\
\end{array}$ & $\begin{array}{l}\text { Posición } \\
2014\end{array}$ & $\begin{array}{c}\text { Índice de } \\
\text { Infraestructura } \\
\text { Año } 2014 \\
\end{array}$ & $\begin{array}{l}\text { Posición } \\
2015\end{array}$ & $\begin{array}{c}\text { Índice de } \\
\text { Infraestructura } \\
\text { Año } 2015 \\
\end{array}$ & $\begin{array}{c}\text { Posición } \\
2016\end{array}$ & $\begin{array}{c}\text { Índice de } \\
\text { Infraestructura } \\
\text { Año } 2016 \\
\end{array}$ \\
\hline $\begin{array}{l}\text { Cund/ } \\
\text { Bogotá }\end{array}$ & 1 & 100 & 1 & 100 & 1 & 100 & 1 & 100 \\
\hline Valle & 3 & 50,6 & 3 & 56,8 & 2 & 37,7 & 2 & 57,9 \\
\hline Antioquia & 2 & 73,6 & 2 & 65,1 & 3 & 35,7 & 3 & 51,2 \\
\hline Nariño & 5 & 45,6 & 4 & 54,4 & 5 & 32,5 & 4 & 50,6 \\
\hline Magdalena & 9 & 37,3 & 7 & 43,7 & 8 & 29,9 & 5 & 48,7 \\
\hline Atlántico & 8 & 40,4 & 10 & 41,6 & 7 & 31,1 & 6 & 47,3 \\
\hline Cesar & 10 & 34,5 & 6 & 48,6 & 4 & 33,7 & 7 & 45,5 \\
\hline Santander & 4 & 45,9 & 8 & 43,4 & 6 & 31,6 & 8 & 43,1 \\
\hline Bolívar & 7 & 42,9 & 5 & 49,0 & 9 & 27,3 & 9 & 39,8 \\
\hline Córdoba & 6 & 45,1 & 9 & 41,9 & 12 & 21,4 & 10 & 37,5 \\
\hline Cauca & 11 & 33,7 & 12 & 36,5 & 13 & 17,8 & 11 & 30,6 \\
\hline Boyacá & 13 & 31,5 & 11 & 39,9 & 11 & 21,9 & 12 & 28,5 \\
\hline Tolima & 12 & 33,1 & 15 & 30,0 & 10 & 22,3 & 13 & 28,5 \\
\hline Chocó & 19 & 18,7 & 14 & 35,1 & 15 & 13,8 & 14 & 25,6 \\
\hline $\begin{array}{c}\text { Norte de } \\
\text { Santander }\end{array}$ & 14 & 27,6 & 19 & 21,0 & 17 & 11,0 & 15 & 22,2 \\
\hline San Andrés & 17 & 22,3 & 13 & 35,3 & 14 & 14,3 & 16 & 21,1 \\
\hline Sucre & 16 & 23,0 & 16 & 26,7 & 22 & 9,2 & 17 & 19,5 \\
\hline Huila & 15 & 26,6 & 17 & 25,6 & 20 & 10,1 & 18 & 16,9 \\
\hline La Guajira & 22 & 13,9 & 25 & 8,7 & 18 & 10,9 & 19 & 14,7 \\
\hline Caldas & 18 & 19,1 & 21 & 17,5 & 23 & 8,7 & 20 & 11,5 \\
\hline Meta & 20 & 16,5 & 20 & 20,3 & 19 & 10,2 & 21 & 11,3 \\
\hline Risaralda & 26 & 6,5 & 29 & 4,6 & 25 & 6,6 & 22 & 11,0 \\
\hline Caquetá & 21 & 13,9 & 22 & 14,3 & 21 & 9,9 & 23 & 9,0 \\
\hline Putumayo & 23 & 11,9 & 18 & 25,6 & 24 & 8,4 & 24 & 8,5 \\
\hline Casanare & 25 & 8,9 & 23 & 11,7 & 27 & 4,3 & 25 & 6,6 \\
\hline Quindío & 24 & 11,3 & 27 & 7,7 & 16 & 12,6 & 26 & 6,1 \\
\hline Amazonas & 29 & 2,3 & 24 & 9,7 & 26 & 4,4 & 27 & 5,7 \\
\hline Arauca & 27 & 3,3 & 32 & 0,0 & 28 & 1,6 & 28 & 3,2 \\
\hline Guainía & 31 & 0,2 & 31 & 1,9 & 29 & 1,1 & 29 & 0,6 \\
\hline Guaviare & 30 & 1,6 & 28 & 5,7 & 32 & 0,0 & 30 & 0,3 \\
\hline Vaupés & 32 & 0,0 & 30 & 3,4 & 31 & 0,4 & 31 & 0,1 \\
\hline Vichada & 28 & 3,1 & 26 & 8,0 & 30 & 0,6 & 32 & 0,0 \\
\hline
\end{tabular}

Fuente: elaboración propia. 
Elcira Solano-Benavides • Nelson De Jesús Alandete-Brochero

Tabla 7. Índice de economía geográfica, 2013-2016

\begin{tabular}{|c|c|c|c|c|c|c|c|c|}
\hline Dep. & $\begin{array}{c}\text { Posición } \\
2013\end{array}$ & $\begin{array}{c}\text { İndice de } \\
\text { Economía } \\
\text { Geográfica } \\
\text { Año } 2013\end{array}$ & $\begin{array}{c}\text { Posición } \\
2014\end{array}$ & $\begin{array}{c}\text { Índice de } \\
\text { Economía } \\
\text { Geográfica } \\
\text { Año } 2014\end{array}$ & $\begin{array}{c}\text { Posición } \\
2015\end{array}$ & $\begin{array}{c}\text { Índice de } \\
\text { Economía } \\
\text { Geográfica } \\
\text { Año } 2015\end{array}$ & $\begin{array}{c}\text { Posición } \\
2016\end{array}$ & $\begin{array}{c}\text { Índice de } \\
\text { Economía } \\
\text { Geográfica } \\
\text { Año } 2016\end{array}$ \\
\hline Atlántico & 1 & 100 & 1 & 100 & 2 & 99,7 & 1 & 100 \\
\hline Sucre & 3 & 94,3 & 2 & 97,3 & 1 & 100 & 2 & 99,7 \\
\hline La Guajira & 5 & 89,3 & 3 & 90,7 & 3 & 93,8 & 3 & 93,9 \\
\hline Cesar & 6 & 84,0 & 6 & 80,0 & 4 & 88,3 & 4 & 92,0 \\
\hline Magdalena & 4 & 92,0 & 4 & 81,8 & 5 & 87,5 & 5 & 90,7 \\
\hline Córdoba & 7 & 81,3 & 7 & 74,8 & 6 & 81,2 & 6 & 86,9 \\
\hline Tolima & 11 & 76,2 & 15 & 62,5 & 8 & 77,3 & 7 & 86,7 \\
\hline Bolívar & 2 & 95,1 & 5 & 80,1 & 7 & 80,7 & 8 & 86,4 \\
\hline Caldas & 8 & 78,1 & 11 & 63,4 & 16 & 71,1 & 9 & 83,5 \\
\hline Valle & 9 & 77,3 & 8 & 66,2 & 12 & 74,2 & 10 & 82,1 \\
\hline $\begin{array}{l}\text { Cund/ } \\
\text { Bogotá }\end{array}$ & 10 & 76,5 & 12 & 63,3 & 14 & 73,3 & 11 & 81,7 \\
\hline Santander & 15 & 68,6 & 13 & 63,0 & 10 & 74,9 & 12 & 81,4 \\
\hline Nariño & 13 & 70,0 & 21 & 54,3 & 21 & 64,7 & 13 & 80,8 \\
\hline Antioquia & 14 & 69,2 & 10 & 63,8 & 11 & 74,3 & 14 & 78,1 \\
\hline Risaralda & 16 & 65,9 & 14 & 62,8 & 15 & 73,1 & 15 & 76,6 \\
\hline Cauca & 12 & 72,2 & 16 & 61,2 & 17 & 70,0 & 16 & 76,4 \\
\hline Boyacá & 21 & 59,3 & 23 & 51,0 & 20 & 65,9 & 17 & 76,0 \\
\hline $\begin{array}{c}\text { Norte de } \\
\text { Santander }\end{array}$ & 19 & 63,0 & 18 & 57,9 & 18 & 68,8 & 18 & 75,6 \\
\hline Quindío & 17 & 65,9 & 9 & 64,2 & 9 & 75,8 & 19 & 75,4 \\
\hline Casanare & 18 & 65,1 & 22 & 53,5 & 22 & 64,2 & 20 & 74,7 \\
\hline Huila & 23 & 57,4 & 19 & 57,0 & 13 & 73,9 & 21 & 73,5 \\
\hline Chocó & 24 & 54,3 & 24 & 39,3 & 24 & 55,7 & 22 & 73,1 \\
\hline Meta & 22 & 59,2 & 20 & 54,6 & 23 & 62,9 & 23 & 71,5 \\
\hline Arauca & 20 & 62,8 & 17 & 60,3 & 19 & 68,6 & 24 & 69,4 \\
\hline Vichada & 25 & 54,2 & 25 & 36,4 & 27 & 33,9 & 25 & 60,1 \\
\hline Putumayo & 27 & 36,9 & 27 & 35,3 & 26 & 48,1 & 26 & 56,0 \\
\hline Caquetá & 26 & 37,5 & 26 & 36,0 & 25 & 49,4 & 27 & 53,5 \\
\hline Guainía & 29 & 15,5 & 31 & 0,0 & 29 & 20,0 & 28 & 29,1 \\
\hline Guaviare & 28 & 19,3 & 28 & 22,3 & 28 & 27,8 & 29 & 23,3 \\
\hline Amazonas & 30 & 8,8 & 30 & 0,4 & 31 & 0,0 & 30 & 7,8 \\
\hline Vaupés & 31 & 0 & 29 & 1,0 & 30 & 0,5 & 31 & 0,0 \\
\hline
\end{tabular}

Fuente: elaboración propia. 
desempeño integral, índice de desempeño fiscal e índice de gobierno abierto, elegidas teniendo en cuenta la teoría de la nueva economía institucional y su nivel de significancia con el PIB real per cápita departamental. Según el análisis de componentes principales, la variable que presenta mayor carga factorial es la de gobierno abierto con 0,924 .

Índice institucional $=f$ (Índice de desempeño integral; Índice de desempeño fiscal e Índice de gobierno abierto) (11)

Los resultados del índice de economía institucional propuesto en el trabajo (Tabla 8), presentan a un $45 \%$ de los departamentos en el nivel alto. En el 2013, en este nivel, se encuentran Quindío, Meta, Boyacá y Santander; los cuales tuvieron un alza coyuntural en los índices de gobierno abierto, desempeño integral y fiscal (Procuraduría General de la Nación, 2013). Pero en los siguientes años bajan de posición y solo Meta y Quindío logran mantenerse entre los cinco primeros puestos. Los departamentos de Cundinamarca/ Bogotá, Antioquia y Risaralda, en tres años han subido y mantenido los primeros puestos gracias especialmente a su desempeño fiscal e integral.

En el nivel medio se encuentra el 34\% de los departamentos y se destacan Bolívar, Magdalena, Caquetá, Arauca, Cesar, Córdoba, Sucre y Cauca.

En el nivel bajo está el $20 \%$ de los departamentos del país y los que se encuentran en los últimos lugares son Vaupés, Guajira, San Andrés, Putumayo, Guainía, Amazonas y Chocó. Estos cuentan con las administraciones locales más débiles en cuanto a la gestión de los recursos públicos.

\subsection{6 Índice capital humano}

El índice capital humano está constituido por las variables de demanda laboral de universitarios $^{6}$ a nivel departamental, cobertura neta

6 Numero de graduados universitarios en el mercado laboral forma que cotiza seguridad social. de educación secundaria, cobertura neta de educación media, puntaje pruebas saber 11 . Variables elegidas teniendo en cuenta la teoría del capital humano y su nivel de significancia con el PIB real per cápita departamental. Según el modelo de componentes principales, la variable que presenta mayor carga factorial es cobertura neta de educación media con 0,986 .

\section{Capital humano $=($ Demanda laboral de}

universitarios; Cobertura neta de educación secundaria; Cobertura neta de educación media; Puntaje pruebas Saber 11) (12)

Los resultados del índice capital humano propuesto en el trabajo (Tabla 9), muestra que el departamento con mayor puntaje es Bogotá/ Cundinamarca, pues esta región tiene la mayor demanda laboral universitaria, según datos del Observatorio Laboral de Educación; además, esta región obtiene los mejores puntajes en las pruebas saber 11 (Barón-Rivera, 2010). En el nivel alto siempre se encuentra Santander, debido a su cobertura en educación básica y media, la cual es uno de las más altas del país según el Departamento Nacional de Planeación (DNP).

En el nivel medio se encuentra el $71 \%$ de los departamentos del país y se destacan Boyacá, Antioquia, Quindío y Atlántico, los cuales poseen altas coberturas en educación secundaria y media.

En el nivel bajo se encuentran, en promedio, el 23\% de los departamentos y se destacan Vaupés, Chocó, Vichada, Guainía, Guajira, Amazonas y Guaviare, territorios con baja cobertura en educación secundaria y media, además de tener bajas demandas laborales de profesionales y bajos puntajes en las pruebas saber 11.

\subsection{7 Índice de ciencia, tecnología e innovación, 2013-2016}

El índice ciencia, tecnología e innovación está constituido por las variables de Inversión en proyectos de investigación, becas de doctorado, becas de maestrías, y solicitudes de patente. Todas elegidas teniendo en cuenta la teoría del 
Elcira Solano-Benavides • Nelson De Jesús Alandete-Brochero

\begin{tabular}{|c|c|c|c|c|c|c|c|c|}
\hline Dep. & $\begin{array}{c}\text { Posición } \\
2013\end{array}$ & $\begin{array}{c}\text { Índice de } \\
\text { Economía } \\
\text { Institucional } \\
\text { Año } 2013 \\
\end{array}$ & $\begin{array}{c}\text { Posición } \\
2014\end{array}$ & $\begin{array}{c}\text { Índice de } \\
\text { Economía } \\
\text { Institucional } \\
\text { Año } 2014\end{array}$ & $\begin{array}{c}\text { Posición } \\
2015\end{array}$ & $\begin{array}{c}\text { Índice de } \\
\text { Economía } \\
\text { Institucional } \\
\text { Año } 2015\end{array}$ & $\begin{array}{l}\text { Posición } \\
2016\end{array}$ & $\begin{array}{c}\text { Índice de } \\
\text { Economía } \\
\text { Institucional } \\
\text { Año } 2016 \\
\end{array}$ \\
\hline $\begin{array}{l}\text { Cund/ } \\
\text { Bogotá }\end{array}$ & 6 & 87,5 & 1 & 100 & 1 & 100 & 1 & 100 \\
\hline Risaralda & 9 & 77,2 & 5 & 93,1 & 3 & 92,3 & 2 & 89,9 \\
\hline Antioquia & 7 & 85,6 & 2 & 99,43 & 2 & 96,1 & 3 & 86,4 \\
\hline Quindío & 1 & 100 & 4 & 95,3 & 6 & 90,0 & 4 & 84,2 \\
\hline Meta & 2 & 99,3 & 3 & 97,6 & 4 & 91,1 & 5 & 83,2 \\
\hline Nariño & 5 & 87,5 & 13 & 78,0 & 7 & 84,9 & 6 & 81,2 \\
\hline Huila & 10 & 75,6 & 6 & 90,2 & 5 & 91,0 & 7 & 79,3 \\
\hline Atlántico & 11 & 75,6 & 7 & 89,2 & 8 & 82,5 & 8 & 77,0 \\
\hline Valle & 12 & 75,3 & 11 & 79,8 & 10 & 80,3 & 9 & 75,0 \\
\hline Casanare & 8 & 83,8 & 8 & 86,6 & 15 & 71,1 & 10 & 72,9 \\
\hline Santander & 4 & 88,6 & 16 & 71,3 & 11 & 79,7 & 11 & 71,2 \\
\hline Boyacá & 3 & 91,5 & 10 & 80,7 & 12 & 78,8 & 12 & 70,6 \\
\hline Sucre & 16 & 67,5 & 24 & 49,4 & 16 & 68,5 & 13 & 68,8 \\
\hline Caldas & 17 & 65,2 & 9 & 83,8 & 9 & 81,2 & 14 & 67,6 \\
\hline $\begin{array}{c}\text { Norte de } \\
\text { Santander }\end{array}$ & 13 & 73,4 & 12 & 78,4 & 14 & 71,5 & 15 & 67,5 \\
\hline Caquetá & 21 & 58,0 & 23 & 50,8 & 22 & 57,9 & 16 & 65,6 \\
\hline Cauca & 15 & 71,7 & 17 & 66,5 & 17 & 68,3 & 17 & 63,9 \\
\hline Magdalena & 22 & 56,3 & 29 & 24,1 & 24 & 48,3 & 18 & 60,8 \\
\hline Arauca & 20 & 60,9 & 15 & 71,4 & 18 & 65,1 & 19 & 59,3 \\
\hline Cesar & 19 & 63,1 & 19 & 57,2 & 21 & 58,6 & 20 & 55,0 \\
\hline Tolima & 14 & 72,9 & 14 & 77,7 & 19 & 63,2 & 21 & 54,5 \\
\hline Bolívar & 23 & 55,2 & 28 & 25,0 & 23 & 49,2 & 22 & 51,0 \\
\hline Córdoba & 18 & 63,4 & 21 & 54,0 & 13 & 73,9 & 23 & 47,8 \\
\hline Vichada & 28 & 22,5 & 22 & 52,1 & 20 & 60,5 & 24 & 46,4 \\
\hline Guaviare & 26 & 30,1 & 20 & 54,1 & 26 & 31,7 & 25 & 46,3 \\
\hline Vaupés & 31 & 17,5 & 27 & 25,2 & 27 & 22,8 & 26 & 41,3 \\
\hline San Andrés & 30 & 18,9 & 30 & 7,3 & 32 & 0,0 & 27 & 27,6 \\
\hline La Guajira & 24 & 48,4 & 18 & 60,5 & 25 & 41,9 & 28 & 26,9 \\
\hline Putumayo & 29 & 19,7 & 26 & 25,4 & 28 & 19,0 & 29 & 26,9 \\
\hline Amazonas & 32 & 0,0 & 25 & 40,8 & 29 & 17,8 & 30 & 18,7 \\
\hline Chocó & 27 & 24,3 & 31 & 4,4 & 31 & 2,2 & 31 & 18,0 \\
\hline Guainía & 25 & 34,6 & 32 & 0,0 & 30 & 14,8 & 32 & 0,0 \\
\hline
\end{tabular}

Fuente: elaboración propia. 
Estimación y comparación de la competitividad regional en Colombia

Tabla 9. Índice de capital humano, 2013-2016

\begin{tabular}{|c|c|c|c|c|c|c|c|c|}
\hline Dep. & $\begin{array}{l}\text { Posición } \\
2013\end{array}$ & $\begin{array}{c}\text { Índice de } \\
\text { Capital } \\
\text { Humano } \\
\text { Año } 2013 \\
\end{array}$ & $\begin{array}{c}\text { Posición } \\
2014\end{array}$ & $\begin{array}{c}\text { Índice de } \\
\text { Capital } \\
\text { Humano } \\
\text { Año } 2014 \\
\end{array}$ & $\begin{array}{l}\text { Posición } \\
2015\end{array}$ & $\begin{array}{c}\text { Índice de } \\
\text { Capital } \\
\text { Humano } \\
\text { Año } 2015 \\
\end{array}$ & $\begin{array}{l}\text { Posición } \\
2016\end{array}$ & $\begin{array}{c}\text { Índice de } \\
\text { Capital } \\
\text { Humano } \\
\text { Año } 2016 \\
\end{array}$ \\
\hline $\begin{array}{l}\text { Cund/ } \\
\text { Bogota }\end{array}$ & 1 & 100 & 1 & 100 & 1 & 100 & 1 & 100 \\
\hline Santander & 2 & 70,5 & 2 & 72,2 & 2 & 75,3 & 2 & 70,4 \\
\hline Boyacá & 3 & 66,1 & 3 & 67,7 & 3 & 69,8 & 3 & 65,8 \\
\hline Antioquia & 4 & 64,5 & 4 & 65,2 & 4 & 67,3 & 4 & 64,1 \\
\hline Quindío & 5 & 63,3 & 5 & 60,7 & 6 & 63,4 & 5 & 63,5 \\
\hline Atlántico & 6 & 61,7 & 8 & 58,4 & 9 & 61,4 & 6 & 61,6 \\
\hline Casanare & 8 & 59,3 & 7 & 59,0 & 5 & 65,6 & 7 & 59,6 \\
\hline Risaralda & 9 & 59,3 & 6 & 59,1 & 8 & 62,7 & 8 & 59,5 \\
\hline Valle & 7 & 60,6 & 9 & 58,0 & 12 & 56,0 & 9 & 59,1 \\
\hline Caldas & 10 & 57,7 & 11 & 56,7 & 10 & 58,1 & 10 & 57,7 \\
\hline Meta & 11 & 56,0 & 10 & 57,3 & 7 & 63,4 & 11 & 56,0 \\
\hline Huila & 12 & 54,0 & 12 & 55,1 & 11 & 57,7 & 12 & 53,9 \\
\hline Tolima & 13 & 51,2 & 14 & 50,4 & 14 & 54,8 & 13 & 51,5 \\
\hline $\begin{array}{c}\text { Norte de } \\
\text { Santander }\end{array}$ & 14 & 50,1 & 13 & 51,4 & 13 & 55,9 & 14 & 49,8 \\
\hline Sucre & 15 & 47,3 & 15 & 47,5 & 16 & 49,4 & 15 & 47,3 \\
\hline Córdoba & 16 & 47,1 & 16 & 44,9 & 17 & 48,7 & 16 & 46,6 \\
\hline Bolívar & 17 & 44,4 & 17 & 44,1 & 18 & 45,4 & 17 & 44,2 \\
\hline Cesar & 18 & 43,9 & 18 & 44,0 & 15 & 49,9 & 18 & 43,8 \\
\hline San Andrés & 19 & 43,4 & 20 & 40,5 & 19 & 45,2 & 19 & 43,6 \\
\hline Nariño & 20 & 40,8 & 19 & 40,9 & 20 & 41,4 & 20 & 41,0 \\
\hline Cauca & 21 & 36,6 & 21 & 36,7 & 22 & 36,6 & 21 & 36,5 \\
\hline Arauca & 22 & 35,6 & 23 & 33,4 & 23 & 36,5 & 22 & 35,8 \\
\hline Putumayo & 23 & 35,5 & 24 & 33,1 & 21 & 37,6 & 23 & 35,6 \\
\hline Magdalena & 24 & 34,2 & 22 & 36,0 & 24 & 36,5 & 24 & 34,0 \\
\hline Caquetá & 25 & 30,3 & 25 & 27,6 & 25 & 30,1 & 25 & 30,4 \\
\hline Guaviare & 26 & 20,4 & 27 & 17,5 & 27 & 20,2 & 26 & 20,5 \\
\hline Amazonas & 27 & 18,7 & 26 & 21,3 & 26 & 21,6 & 27 & 18,7 \\
\hline La Guajira & 28 & 17,3 & 28 & 16,0 & 28 & 16,2 & 28 & 17,2 \\
\hline Guainía & 29 & 12,4 & 29 & 13,2 & 31 & 4,7 & 29 & 12,4 \\
\hline Vichada & 30 & 9,9 & 31 & 6,4 & 29 & 9,2 & 30 & 9,9 \\
\hline Chocó & 31 & 8,2 & 30 & 7,3 & 30 & 8,0 & 31 & 8,1 \\
\hline Vaupés & 32 & 0,0 & 32 & 0,0 & 32 & 0,0 & 32 & 0,0 \\
\hline
\end{tabular}

Fuente: elaboración propia. 
capital humano y su nivel de significancia con el PIB real per cápita departamental. Según el modelo de componentes principales la variable que presenta mayor carga factorial es solicitudes de patente con 0,969 .

\section{Ciencia, tecnología e innovación $=f$ (Inversión en proyectos de investigación; Becas de doctorado asignadas, Becas de maestrías asignadas; Solicitudes de patente) (13)}

Los resultados del índice ciencia, tecnología e innovación propuesto en el trabajo (Tabla 10), muestra que el departamento con mayor indicador en el periodo de estudio y con gran diferencia de los demás es Cundinamarca/Bogotá, este primer puesto se debe a la concentración de empresas, centros de investigaciones y universidades que son las instituciones que promueven los proyectos de investigación y solicitudes de patentes. En el indicador se observa una gran brecha regional entre Cundinamarca/Bogotá y el resto de departamentos en Colombia.

De igual manera, Antioquia, es el único departamento en nivel medio gracias a que es la segunda en aglomeración del país, algo que le da ventajas al tener una alta densidad empresarial y un número de universidades que incentivan el desarrollo de investigación e innovación.

En el nivel bajo se encuentran los otros 30 departamentos, es decir, cerca del 93\%; se destacan Valle, Santander, Caldas, Boyacá y Atlántico en los primeros lugares. En contraste, Vichada, Arauca, San Andrés, Casanare, Guaviare, Putumayo y Guajira se encuentran en los últimos lugares.

\subsection{8 Índice regional de competitividad departamental (IRCD), 2013-2016}

El índice regional de competitividad departamental (IRCD) está constituido por los índices estimados de aglomeración, fuerzas centrífugas, infraestructura, ciencia y tecnología, economía geográfica, economía institucional y capital humano; los cuales son coherentes con la competitividad regional que se enmarca en las teorías de aglomeraciones, economía geográfica, economía institucional y capital humano. Así mismo, estos índices están relacionados con el PIB real per cápita de los departamentos. Según el análisis de compontes principales el indicador que presenta mayor carga factorial, es decir, que tiene mayor peso es Ciencia y Tecnología con 0,970.

Los resultados del índice regional de competitividad departamental, propuesto en el trabajo (Tabla 11), muestran a Cundinamarca/Bogotá como líder en todos los años y es el único en el nivel alto. Esto se debe a su liderazgo en cinco de los sietes indicadores que son aglomeración, infraestructura, economía institucional, capital humano y ciencia y tecnología.

En el nivel medio se encuentran Antioquia, Valle y Santander, que representan solo el $9 \%$ de los departamentos en Colombia, además son las regiones con mayores aportes al producto nacional.

En el nivel bajo se encuentra el $87 \%$ de los departamentos en Colombia, revelando una desigualdad en términos de competitividad explicada por los factores de aglomerados, infraestructura, economía institucional, capital humano y ciencia y tecnología. Entre las mejores posiciones del nivel están Atlántico, Boyacá, Bolívar y Nariño. Por otro lado, los departamentos en los puestos más bajos pertenecen a las regiones de la Amazonía y la Orinoquía: Amazonas, Guaviare, Vichada, Guainía, Putumayo y Vaupés.

\section{Evaluación del índice regional de competitividad departamental (IRCD) con el PIB per cápita departamental}

Con el fin de evaluar el indicador aquí propuesto de la misma manera que se hizo con los indicadores de la CEPAL y el CPC, se estima un modelo econométrico panel data con el PIB per cápita real departamental y los siete indicadores de competitividad estimados con el modelo de componentes principales (ecuación 14): aglomeración, fuerzas centrifugas, Capital humano, 
Estimación y comparación de la competitividad regional en Colombia

Tabla 10. Índice de Ciencia, Tecnología e Innovación, 2013-2016

\begin{tabular}{|c|c|c|c|c|c|c|c|c|}
\hline Dep. & $\begin{array}{l}\text { Posición } \\
2013\end{array}$ & $\begin{array}{c}\text { İndice de } \\
\text { Ciencia y } \\
\text { Tecnología } \\
\text { Año } 2013\end{array}$ & $\begin{array}{c}\text { Posición } \\
2014\end{array}$ & $\begin{array}{c}\text { Índice de } \\
\text { Ciencia y } \\
\text { Tecnología } \\
\text { Año } 2014\end{array}$ & $\begin{array}{c}\text { Posición } \\
2015\end{array}$ & $\begin{array}{c}\text { Índice de } \\
\text { Ciencia y } \\
\text { Tecnología } \\
\text { Año } 2015\end{array}$ & $\begin{array}{l}\text { Posición } \\
2016\end{array}$ & $\begin{array}{c}\text { Índice de } \\
\text { Ciencia y } \\
\text { Tecnología } \\
\text { Año } 2016\end{array}$ \\
\hline $\begin{array}{l}\text { Cund/ } \\
\text { Bogotá }\end{array}$ & 1 & 100 & 1 & 100 & 1 & 100 & 1 & 100 \\
\hline Antioquia & 2 & 53,5 & 2 & 49,5 & 2 & 57,03 & 2 & 43,11 \\
\hline Valle & 3 & 21,0 & 3 & 19,9 & 3 & 22,46 & 3 & 35,35 \\
\hline Santander & 4 & 19,0 & 4 & 17,1 & 5 & 16,10 & 4 & 21,11 \\
\hline $\begin{array}{c}\text { Norte de } \\
\text { Santander }\end{array}$ & 15 & 1,5 & 17 & 2,8 & 15 & 2,22 & 5 & 14,97 \\
\hline Atlántico & 5 & 10,8 & 5 & 13,5 & 6 & 9,04 & 6 & 12,15 \\
\hline Tolima & 9 & 3,4 & 15 & 3,1 & 13 & 3,35 & 7 & 9,87 \\
\hline Caldas & 6 & 5,2 & 6 & 6,5 & 7 & 5,72 & 8 & 7,22 \\
\hline Nariño & 8 & 3,5 & 14 & 3,8 & 10 & 3,96 & 9 & 6,91 \\
\hline Boyacá & 7 & 4,4 & 11 & 4,7 & 4 & 17,82 & 10 & 6,60 \\
\hline Quindío & 13 & 2,4 & 20 & 1,8 & 11 & 3,72 & 11 & 5,02 \\
\hline Risaralda & 11 & 3,1 & 10 & 5,0 & 12 & 3,40 & 12 & 4,95 \\
\hline Bolívar & 12 & 2,7 & 9 & 5,1 & 8 & 4,64 & 13 & 4,85 \\
\hline Putumayo & 24 & 0,4 & 23 & 0,4 & 28 & 0,11 & 14 & 4,28 \\
\hline Huila & 14 & 1,7 & 16 & 2,9 & 20 & 1,54 & 15 & 3,32 \\
\hline Cauca & 10 & 3,4 & 8 & 5,2 & 9 & 4,27 & 16 & 1,85 \\
\hline Córdoba & 17 & 1,2 & 19 & 2,0 & 17 & 2,20 & 17 & 1,79 \\
\hline Meta & 18 & 1,1 & 21 & 1,1 & 18 & 1,82 & 18 & 1,77 \\
\hline Cesar & 20 & 0,8 & 13 & 4,0 & 21 & 0,94 & 19 & 1,62 \\
\hline Magdalena & 22 & 0,6 & 7 & 5,7 & 14 & 2,29 & 20 & 1,50 \\
\hline Sucre & 25 & 0,3 & 18 & 2,5 & 19 & 1,64 & 21 & 1,40 \\
\hline Caquetá & 21 & 0,7 & 24 & 0,3 & 22 & 0,64 & 22 & 0,78 \\
\hline La Guajira & 23 & 0,5 & 22 & 0,4 & 24 & 0,30 & 23 & 0,57 \\
\hline Guainía & 30 & 0,0 & 29 & 0,1 & 30 & 0,04 & 24 & 0,22 \\
\hline Casanare & 27 & 0,2 & 26 & 0,2 & 27 & 0,18 & 25 & 0,19 \\
\hline Arauca & 28 & 0,1 & 28 & 0,2 & 26 & 0,21 & 26 & 0,11 \\
\hline San Andrés & 29 & 0,1 & 27 & 0,2 & 23 & 0,50 & 27 & 0,11 \\
\hline Amazonas & 16 & 1,2 & 30 & 0,0 & 29 & 0,04 & 28 & 0,06 \\
\hline Chocó & 19 & 1,0 & 12 & 4,1 & 16 & 2,21 & 29 & 0,06 \\
\hline Guaviare & 26 & 0,3 & 25 & 0,3 & 25 & 0,26 & 30 & 0,0 \\
\hline Vaupés & 31 & 0,0 & 31 & 0,0 & 31 & 0,0 & 31 & 0,0 \\
\hline Vichada & 32 & 0,0 & 32 & 0,0 & 32 & 0,0 & 32 & 0,0 \\
\hline
\end{tabular}

Fuente: elaboración propia. 
Elcira Solano-Benavides • Nelson De Jesús Alandete-Brochero

Tabla 11. Índice Regional de Competitividad Departamental, 2013-2016

\begin{tabular}{|c|c|c|c|c|c|c|c|c|}
\hline Dep. & $\begin{array}{c}\text { Posición } \\
2013\end{array}$ & $\begin{array}{c}\text { IRCD } \\
\text { año } 2013\end{array}$ & $\begin{array}{c}\text { Posición } \\
2014\end{array}$ & $\begin{array}{c}\text { IRCD año } \\
2014\end{array}$ & $\begin{array}{c}\text { Posición } \\
2015\end{array}$ & $\begin{array}{c}\text { IRCD } \\
\text { año } 2015\end{array}$ & $\begin{array}{c}\text { Posición } \\
2016\end{array}$ & $\begin{array}{c}\text { IRCD } \\
\text { año } 2016\end{array}$ \\
\hline $\begin{array}{l}\text { Cund/ } \\
\text { Bogotá }\end{array}$ & 1 & 100 & 1 & 100 & 1 & 100 & 1 & 100 \\
\hline Antioquia & 2 & 57,0 & 2 & 55,1 & 2 & 50,3 & 2 & 48,0 \\
\hline Valle & 3 & 35,3 & 3 & 37,6 & 3 & 34,8 & 3 & 42,1 \\
\hline Santander & 4 & 32,2 & 4 & 33,6 & 4 & 29,3 & 4 & 32,3 \\
\hline Atlántico & 5 & 21,7 & 5 & 24,1 & 6 & 22,4 & 5 & 24,8 \\
\hline Nariño & 8 & 15,8 & 8 & 20,1 & 9 & 15,7 & 6 & 19,6 \\
\hline Bolívar & 6 & 18,8 & 6 & 22,7 & 8 & 15,8 & 7 & 18,5 \\
\hline Boyacá & 7 & 17,9 & 7 & 22,4 & 5 & 22,8 & 8 & 17,6 \\
\hline $\begin{array}{c}\text { Norte de } \\
\text { Santander }\end{array}$ & 15 & 12,3 & 18 & 12,4 & 18 & 10,8 & 9 & 16,7 \\
\hline Tolima & 10 & 15,1 & 13 & 16,3 & 7 & 15,9 & 10 & 15,5 \\
\hline Magdalena & 17 & 11,4 & 10 & 16,9 & 14 & 11,6 & 11 & 14,1 \\
\hline Cesar & 14 & 12,5 & 9 & 19,0 & 11 & 15,2 & 12 & 13,7 \\
\hline Córdoba & 9 & 15,5 & 11 & 16,7 & 16 & 11,3 & 13 & 13,6 \\
\hline Risaralda & 20 & 8,7 & 21 & 9,6 & 19 & 10,5 & 14 & 12,8 \\
\hline Cauca & 13 & 12,6 & 14 & 15,5 & 15 & 11,5 & 15 & 12,7 \\
\hline Huila & 12 & 13,1 & 15 & 14,7 & 20 & 10,3 & 16 & 12,3 \\
\hline Caldas & 16 & 11,9 & 17 & 13,2 & 13 & 11,7 & 17 & 12,0 \\
\hline San Andrés & 18 & 11,0 & 16 & 14,0 & 17 & 10,9 & 18 & 11,1 \\
\hline Quindío & 19 & 8,9 & 24 & 8,7 & 12 & 11,9 & 19 & 11,1 \\
\hline Meta & 11 & 13,4 & 12 & 16,3 & 10 & 15,6 & 20 & 10,1 \\
\hline Sucre & 21 & 8,5 & 19 & 11,5 & 24 & 6,1 & 21 & 8,9 \\
\hline Casanare & 22 & 8,2 & 20 & 10,4 & 21 & 10,2 & 22 & 7,8 \\
\hline Caquetá & 24 & 6,0 & 25 & 6,1 & 25 & 5,5 & 23 & 6,5 \\
\hline Putumayo & 23 & 6,0 & 23 & 9,0 & 27 & 4,9 & 24 & 6,3 \\
\hline Amazonas & 28 & 3,1 & 26 & 4,2 & 23 & 6,9 & 25 & 4,4 \\
\hline Chocó & 26 & 3,9 & 22 & 9,4 & 29 & 3,0 & 26 & 4,2 \\
\hline Arauca & 27 & 3,5 & 28 & 3,2 & 26 & 5,2 & 27 & 3,8 \\
\hline Guaviare & 29 & 2,2 & 29 & 2,4 & 28 & 4,2 & 28 & 3,7 \\
\hline Vaupés & 32 & 0 & 32 & 0 & 31 & 2,2 & 29 & 2,4 \\
\hline La Guajira & 25 & 4,0 & 27 & 3,8 & 22 & 7,2 & 30 & 1,9 \\
\hline Guainía & 30 & 1,1 & 30 & 1,9 & 32 & 0 & 31 & 0,4 \\
\hline Vichada & 31 & 0,3 & 31 & 1,7 & 30 & 2,4 & 32 & 0 \\
\hline
\end{tabular}

Fuente: elaboración propia. 
Economía institucional, Infraestructura, Geografía económica y Ciencia y tecnología, para los años 2013, 2014, 2015 y 2016.

El modelo panel data se especifica de la siguiente manera:

$$
\begin{gathered}
Y_{i t}=v_{i}+\beta_{1} \text { Aglomeración }_{i t}+\beta_{2} \text { Fuerza Centrifuga }_{i t}+ \\
\beta_{3} \text { Capital Humano }_{i t}+\beta_{4} \text { Institucional }_{i t}{ }^{+} \\
\beta_{5} \text { Infraestructura }_{i t}+\beta_{6} \text { Geografía Económica }_{i t}+ \\
\beta_{7} \text { Ciencias y Tecnología }^{+} e_{i t}(14)
\end{gathered}
$$

Donde $Y_{i t}=$ es el logaritmo del PIB real per cápita del departamento i en el año $t ; v_{i}$ es un vector de variables dicotómicas para cada departamento $i ; \beta_{1 . .7}=$ son los coeficientes de los índices regionales de competitividad; $y e_{i t}$ es el error aleatorio.

\subsection{Los resultados de la evaluación de índice regional de competitividad departamental}

Como se observa en la Tabla 12, todos los índices de competitividad regional departamental estimados en este proyecto de investigación son significativos estadísticamente con el PIB per cápita departamental y tienen un margen de error del 10\%; los índices positivos son: fuerzas centrípetas $(0,0261662)$, fuerzas centrifugas $(0,0042219)$, capital humano $(0,0031687)$, ciencia, tecnología e innovación $(0,0031631)$ e instituciones $(0,000768)$. Es decir, cuando los índices de fuerzas centrípetas y fuerzas centrífugas aumentan en una unidad, el nivel de productividad departamental crece en $0,02 \%$ y $0,004 \%$ respectivamente. De igual manera, los índices de competitividad regional departamental impactan positivamente el PIB per cápita, que es un indicador de bienestar económico.

Los índices de competitividad regional departamental significativos y con signo negativo son: infraestructura $(-0,001007)$ y geografía económica $(-0,0002062)$. Es decir, los índices de infraestructura y geografía económica de los departamentos en Colombia son estadísticamente significativos y negativos con el PIB per cápita, intuitivamente pareciera que la inversión en infraestructura en Colombia no es eficiente para los departamentos.

Tabla 12. Resultado modelo panel data: PIB per cápita con el IRCD, 2013-2014-2015-2016

\begin{tabular}{cccc}
\hline \multicolumn{4}{c}{ Modelo ln PIB real per cápita con el IRCD } \\
\hline Índice & Coeficiente & Std. Err. & P >z \\
\hline Aglomeración & 0,0261662 & 0,0067509 & 0,000 \\
\hline Fuerzas Centrifuga & 0,0042219 & 0,0008739 & 0,000 \\
\hline Capital Humano & 0,0031687 & 0,0017347 & 0,068 \\
\hline Ciencia y tecnología & 0,0031631 & 0,0008875 & 0,000 \\
\hline Economía institucional & 0,000768 & 0,0003413 & 0,024 \\
\hline Economía Geográfica & $-0,0002062$ & 0,0001128 & 0,067 \\
\hline Infraestructura & $-0,001007$ & 0,0004084 & 0,014 \\
\hline
\end{tabular}

Fuente: elaboración propia. 


\section{Discusión de los resultados}

Cuando se comparan los tres indicadores de competitividad regional departamental (CEPAL, PCP y el IRCD) propuestos en esta investigación se encuentran algunas diferencias.

Por ejemplo, las variables de los índices de competitividad propuestos en el trabajo, están sustentados en teorías económicas. Además, la selección de las variables no fue a priori, es el modelo de componentes principales con sus test el que determina su escogencia, asimismo debían pasar el test de significancia en los modelos panel data con el PIB per cápita.

De igual manera, únicamente se utilizaron 26 variables para la construcción de siete indicadores de competitividad regional departamental. En contraste, la CEPAL utilizó 71 variables para cinco índices y el CPC utilizó 97 variables para 10 índices. La utilidad de la reducción de variables es que hace más entendibles los índices desde la teoría económica, y facilita el quehacer de la política económica territorial departamental, determinando las capacidades económicas de cada departamento.

Además, el indicador regional de competitividad departamental propuesto en algunos departamentos cambia en el periodo de estudio, indicando que las políticas de los gobiernos y los comportamientos de los agentes económicos territoriales están incidiendo en el IRCD y que es posible para los departamentos cambiar de posiciones en el tiempo; incidencia que no ocurre con los de la CEPAL y el CPC (ver Tabla 13).

Por último, no todos los factores de los indicadores de competitividad departamental del CPC son significativos con el PIB per cápita, excepto el de ciencia y tecnología. Y para la CEPAL, todos los factores salieron significativos con el PIB per cápita, excepto infraestructura. En contraste, en el IRCD propuesto en este trabajo, todos los índices son significativos en el modelo panel data con la productividad, infiriendo la relación del índice con el PIB per cápita.

\section{Conclusiones}

El índice de competitividad regional departamental para Colombia, propuesto en el trabajo, está compuesto por factores sustentados en las teorías económicas de aglomeraciones, economía geográfica, economía institucional y capital humano. Además, se seleccionaron las variables de los índices de competitividad de manera objetiva, con la utilización del modelo econométrico de componentes principales y panel data para verificar que las variables estén estadísticamente relacionadas con PIB per cápita.

El indicador propuesto en el trabajo presentó siete índices y 26 variables que pueden proveer información útil para la formulación de políticas públicas departamentales.

El índice de aglomeración tiene en la participación del PIB departamental una carga factorial de 0,997, es decir con la mayor representatividad entre las variables. Por tal razón, las políticas económicas territoriales deben estar orientadas a fortalecer las estructuras económicas, por ejemplo los clústeres industriales que desarrollen las relaciones comerciales e industriales complementarias entre los departamentos.

El índice de fuerzas centrifugas tiene en la participación del PIB minero una carga factorial de 0,754 , es decir con la mayor representatividad entre las variables. Tal factor muestra un dinamismo entre los departamentos por el aumento de la producción minera y agrícola. La política pública en estos departamentos debería focalizase en la implementación y el aprovechamiento de las regalías. Esto con la generación de proyectos eficientes en infraestructura y capital humano que puedan impulsar a los departamentos en los otros índices de competitividad.

El índice de infraestructura tiene en la inversión en minas y energía una carga factorial de 0,908 , es decir con la mayor representatividad entre las variables. Además, en este factor, las fuerzas de aglomeración y centrifugas juegan un papel preponderante en la localización de la inversión en Colombia. Tal indicador puede ser guía para la política pública en la inversión 
Tabla 13. Indicadores de competitividad departamental en Colombia

\begin{tabular}{|c|c|c|c|c|c|}
\hline Departamentos & $\begin{array}{l}\text { Posiciones } \\
\text { CEPAL } 2015\end{array}$ & Departamentos & $\begin{array}{c}\text { Posiciones } \\
\text { CPC } 2015\end{array}$ & Departamentos & $\begin{array}{l}\text { Posiciones } \\
\text { IRCD } 2015\end{array}$ \\
\hline Bog-Cund. & 1 & Bogotá, D.C. & 1 & Cund-Bogotá & 1 \\
\hline Antioquia & 2 & Antioquia & 2 & Antioquia & 2 \\
\hline Caldas & 3 & Caldas & 3 & Valle & 3 \\
\hline Santander & 4 & Santander & 4 & Santander & 4 \\
\hline Risaralda & 5 & Valle Del Cauca & 5 & Boyacá & 5 \\
\hline Valle & 6 & Cundinamarca & 6 & Atlántico & 6 \\
\hline Atlántico & 7 & Risaralda & 7 & Tolima & 7 \\
\hline Quindío & 8 & Boyacá & 8 & Bolívar & 8 \\
\hline Boyacá & 9 & Atlántico & 9 & Nariño & 9 \\
\hline Meta & 10 & Quindío & 10 & Meta & 10 \\
\hline Bolívar & 11 & Bolívar & 11 & Cesar & 11 \\
\hline Tolima & 12 & Meta & 12 & Quindío & 12 \\
\hline $\mathrm{N}$ de Santander & 13 & Casanare & 13 & Caldas & 13 \\
\hline Huila & 14 & Cauca & 14 & Magdalena & 14 \\
\hline San Andrés & 15 & Tolima & 15 & Cauca & 15 \\
\hline Casanare & 16 & Huila & 16 & Córdoba & 16 \\
\hline Cesar & 17 & Córdoba & 17 & San Andrés & 17 \\
\hline Magdalena & 18 & N. de Santander & 18 & N. de Santander & 18 \\
\hline Nariño & 19 & Sucre & 19 & Risaralda & 19 \\
\hline Cauca & 20 & Cesar & 20 & Huila & 20 \\
\hline Sucre & 21 & Nariño & 21 & Casanare & 21 \\
\hline Córdoba & 22 & Magdalena & 22 & La Guajira & 22 \\
\hline Caquetá & 23 & Caquetá & 23 & Amazonas & 23 \\
\hline La Guajira & 24 & La Guajira & 24 & Sucre & 24 \\
\hline Arauca & 25 & Putumayo & 25 & Caquetá & 25 \\
\hline Amazonas & 26 & Chocó & 26 & Arauca & 26 \\
\hline Putumayo & 27 & & & Putumayo & 27 \\
\hline Choco & 28 & & & Guaviare & 28 \\
\hline Guaviare & 29 & & & Chocó & 29 \\
\hline Vichada & 30 & & & Vichada & 30 \\
\hline Guainía & 31 & & & Vaupés & 31 \\
\hline Vaupés & 32 & & & Guainía & 32 \\
\hline
\end{tabular}

Fuente: elaboración propia, CEPAL y CPC (2017). 
orientada en mejorar los servicios públicos; algo que ayuda a elevar la calidad de vida de las personas. Además, la inversión debe focalizarse en departamentos con altos potenciales agrícolas y mineros como la región de la Amazonía, y en las regiones orientadas al comercio internacional como la Pacífica y la Caribe.

El índice de economía geográfica tiene en la distancia a puertos marítimos una carga factorial de 0,902 , es decir con la mayor representatividad entre las variables. El indicador sugiere que las políticas públicas deben estar orientadas, en estos departamentos, al comercio internacional con incentivos para la creación de empresas exportadoras, especialmente hacia los departamentos con puertos marítimos. Además, con el mejoramiento logístico de los puertos marítimos de las regiones Caribe y Pacífica, que permitan una diminución de los costos de transporte en términos monetarios y de tiempo. Así mismo, buscar que mediante la integración entre el sector público y el privado se realicen inversiones en infraestructura que permitan el aumento de la capacidad instalada de los puertos, con el fin de aumentar el flujo comercial en el país.

El índice de economía institucional tiene en el gobierno abierto una carga factorial de 0,924, es decir con la mayor representatividad entre las variables. El indicador sugiere que la política pública debería estar orientada a la transparencia, participación ciudadana y lucha contra la corrupción, mediante el control de los órganos estatales y la creación de leyes que incentiven la entrega de información oportuna y actual de las gestiones políticas. Así como a la creación de organizaciones o grupos ciudadanos que defiendan y vigilen los derechos de sus comunidades, del ciudadano y del consumidor. Lo anterior con el fin de crear entornos institucionales que fomenten la competitividad, ayuden a disminuir los costos de transacción y la corrupción.

El índice de capital humano tiene en la cobertura neta en educación media una carga factorial de 0,986, es decir con la mayor representatividad entre las variables. El indicador muestra la importancia de la educación media para la competitividad de los departamentos. Por tal razón, las políticas en torno al capital humano deben estar orientadas a aumentar y mejorar la calidad en la educación media, especialmente en los sectores rurales, mediante la creación de nuevos colegios, inversión en el mejoramiento de la infraestructura educativa de los colegios públicos y creación de servicios de alimentación, donde los jóvenes tengan las condiciones óptimas para cualificarse y mejorar su calidad de vida.

El índice de ciencia, tecnología e innovación tiene en las solicitudes de patentes una carga factorial de 0,969, es decir con la mayor representatividad entre las variables. El índice presenta una alta desigualdad en términos científicos por la concentración de universidades en las principales ciudades del país. Por tal razón, las políticas públicas en ciencia y tecnología deben tener un enfoque regional mediante convocatorias de fortalecimiento de universidades y formación de alto capital humano en los departamentos; y con la formulación y ejecución de proyectos de investigación e innovación que beneficien a la comunidad académica, empresarial y al gobierno en cada departamento.

\section{Referencias bibliográficas}

Acosta, K. D. (2012). Cartagena, entre el progreso industrial y el rezago social. Documentos de Trabajo Sobre Economía Regional y Urbana nº 178. Bogotá, Colombia: Banco de la Republica.

Barón-Rivera, J. D. (2010). La brecha de rendimiento académico de Barranquilla. Documentos de Trabajo Sobre Economía Regional y Urbana n 137. Bogotá, Colombia: Banco de la República.

Begg, I. (2002). Urban Competitiveness, Policies for Dynamic Cities. Bristol, Reino Unido: Policy Press. 
Belderbos, R. y Carree, M. (2002). The location of Japanese investments in China: Agglomeration effects, keiretsu, and firm heterogeneity. Journal of the Japanese and internacional economies, 16(2), 194-211. https://doi.org/10.1006/jiie.2001.0491

Bonet, J. (2007). ¿Por qué es pobre el Chocó? Bogotá, Colombia: Banco de la Republica.

Botero, S. L. (2011). Hacia un nuevo debate sobre la descentralización en Colombia: el análisis intergubernamental desde una lectura institucional. Revista CS, (8), 211-243. https://doi. org/10.18046/recs.18.1134

Cabrero, E., Orihuela, I. y Ziccardi, A. (2003). Ciudades competitivas-ciudades cooperativas: conceptos claves y construcción de un índice para ciudades mexicanas. Ciudad de México, México: CIDE.

Chamorro, M. V., Ortíz, E. V. y Viana, L. A. (2015). Cuantificación y caracterización de la radiación solar en el departamento de la guajira-Colombia mediante el cálculo de transmisibilidad atmosférica. Prospectiva, 13(2), 54-63. https://doi.org/10.15665/rp.v13i2.487

CID -Centro de Investigaciones para el Desarrollo-. (2002). Sistema de indicadores de competitividad departamental SICD. Bogotá, Colombia: CID.

Cohen, B. (2006). Urbanization in developing countries: Current trends, future projections, and key challenges for sustainability. Technology in society, 28(1-2), 63-80. https://doi.org/10.1016/j. techsoc.2005.10.005

CPC -Consejo Privado de Competitividad-. (2013). Índice Departamental de Competitividad. 2013. Bogotá, Colombia: CPC.

CPC -Consejo Privado de Competitividad-. (2014). Índice Departamental de Competitividad. 2014. Bogotá, Colombia: CPC.

CPC -Consejo Privado de Competitividad-. (2015). Índice Departamental de Competitividad. 2015. Bogotá, Colombia: CPC.

CPC -Consejo Privado de Competitividad-. (2016). Índice Departamental de Competitividad. 2016. Bogotá, Colombia: CPC.

CPC -Consejo Privado de Competitividad-. (2017). Base de datos Índice Departamental de Competitividad. Recuperado de: https://compite.com.co/indice-departamental-de-competitividad/

CRECE -Centro de Estudios Regionales Cafeteros y Empresariales-. (2002). Ranking de competitividad de los departamentos colombianos. Manizales, Caldas: CRECE.

Espinosa, A. y Albis, N. (2005). Diagnóstico de la competitividad de Cartagena: La situación de la ciudad a principios del siglo XXI. Cartagena, Colombia: Observatorio del Caribe Colombiano.

FEM -Foro Económico Mundial-. (2014). Global Competitiveness Index (2014-2015). Recuperado de http://www3.weforum.org/docs/WEF_GlobalCompetitivenessReport_2014-15.pdf

Fujita, M., Krugman, P. y Venables, A. (1999). The Spatial Economy: Cities, Regions and International Trade. Cambridge, Estados Unidos: The MIT Press.

Galvis, L. A. (2014). Economía de las grandes ciudades en Colombia: seis estudios de caso. Bogotá, Colombia: Banco de la República. 
Hall, R. E. y Jones, C. I. (1999). Why do some countries produce so much more output per worker than others? The quarterly journal of economics, 114(1), 83-116. https://doi.org/10.3386/w6564

Heckscher, E. F. (1919). Heckscher-Ohlin trade theory. Cambridge, Estados Unidos: MIT press.

Huber, G. y Mungaray, A. (2017). Los índices de competitividad en México. Gestión y política pública, 26(1), 167-218. https://doi.org/10.29265/gypp.v26il.216

Huovari, J., Kangasharju, A. y Alanen, A. (2001). Constructing an index for regional competitiveness. En Z. Acs, H. de Groot y P. Nijkamp (Eds.), The Emergence of the Knowledge Economy (pp. 121-138). Berlín, Alemania: Springer.

Instituto para la Gestión del Desarrollo. (1996). IMD World Competitiveness Yearbook. Lausanne, Suiza: IMD World competitiveness center.

Jain, A., Nandakumar, K. y Ross, A. (2005). Score normalization in multimodal biometric systems. Pattern recognition, 38(12), 2270-2285. https://doi.org/10.1016/j.patcog.2005.01.012

Krugman, P. (1988). La nueva teoría del comercio internacional y los países menos desarrollados. El trimestre económico, 55(217), 41-66.

Krugman, P. (1995). Competitividad: una peligrosa obsesión. Ensayos de Economía, 6(9-10), 17-34.

Krugman, P. (1997). Desarrollo, geografía y teoría económica. Barcelona, España: Antoni Bosch.

Krugman, P. y Wells, R. (2006). Introducción a la Economía: Microeconomía. Barcelona, España: Editorial Reverté.

Lall, S. (2001). Comparing national competitive performance: An economic analysis of World Economic Forum's competitiveness index. Oxford, Reino Unido: Universidad de Oxford.

Lotero, J. (2007). Desarrollo y organización espacial de la industria manufacturera colombiana: algunas implicaciones para la competitividad regional. En J. Lotero (Ed.), Industria y Región en Colombia (pp. 19-101). Medellín, Colombia: Universidad de Antioquia.

Lotero, J., Posada, H. M. y Valderrama, D. (2009). La competitividad de los departamentos colombianos desde la perspectiva de la geografía económica. Lecturas de economía, (71), 107-139.

Lozano, A., y Yoshida, P. (2008). Índice de competitividad regional cafetero. Revista Ensayos sobre economía cafetera, (24), 103-131.

Lucas, R. E. (1988). On the mechanics of economic development. Journal of monetary economics, 22(1), 3-42. https://doi.org/10.1016/0304-3932(88)90168-7

Manrique, O.L.(2006).Fuentes de las economías de aglomeración: una revisión bibliográfica.Cuadernos de economía, 25(45), 75-93.

Martin, R. L. (2003). A study on the factors of regional competitiveness. Cambridge, Reino Unido: University of Cambridge.

Meisel, A., Bonilla, L. y Sánchez, A. M. (2013). Geografía económica de la Amazonía colombiana. Cartagena, Colombia: Banco de la República.

Musik, G. y Romo, D. (2004). Sobre el concepto de competitividad. Comercio exterior, 55(3), 200-214. 
Nava, R. M., Cernas, D. A. y Becerril, O. U. (2017). Indicador de competitividad municipal en el Estado de México para construir un entorno competitivo. Economía, sociedad y territorio, 17(54), 241-278. https://doi.org/10.22136/esto02017635

Newman P. V. y Ángel, M. P. (2017). Estado del arte sobre la corrupción en Colombia. Bogotá, Colombia: Fedesarrollo.

Ohlin, B. (1935). Interregional and international trade. Cambridge, Estados Unidos: Harvard University Press.

Pérez, G. J. (2005). La infraestructura del transporte vial y la movilización de carga en Colombia. Cartagena, Colombia: Banco de la República.

Porter, M. (1990). The Competitive Advantage of Nations. Nueva York, Estados Unidos: Free Press.

Porter, M. E. (1998). Clusters. Innovation, and Competitiveness: New Findings and Implications for Policy. Recuperado de https://www.academia.edu/2917867/ Clusters_Innovation_and_Competitiveness_New_Findings_and_Implications_for_Policy

Procuraduría General de la Nación. (2013). Herramientas de Fortalecimiento Preventivo y Promoción de la Transparencia, Índice De Gobierno Abierto: Análisis De Su Aplicación. Bogotá, Colombia: Procuraduría General de la Nación.

Ramírez, J. C. y de Aguas, J. M. (2015). Escalafón de la competitividad de los departamentos de Colombia 2015. Santiago, Chile: CEPAL.

Ramírez, J. J. y Parra, R. I. (2009). Escalafón de la competitividad de los departamentos en Colombia 2006. Bogotá, Colombia: Naciones Unidas.

Ramírez, J. C. y Parra, R. I. (2010). Escalafón de la competitividad de los departamentos en Colombia a 2009. Bogotá, Colombia: Naciones Unidas.

Ramírez, J. C., Osorio, H y Parra, R. I. (2007). Escalafón de la competitividad de los departamentos en Colombia. Bogotá, Colombia: Naciones Unidas.

Ramírez, J., Parra, R., González, L. y Corredor, A. (2014). Escalafón de la competitividad de los departamentos de Colombia, 2012. Santiago, Chile: CEPAL.

Ricardo, D. (1817). On the Principles of Political Economy and Taxation. Oxford, Reino Unido: Universidad de Oxford.

Romer, P. M. (1986). Increasing returns and long-run growth. Journal of political economy, 94(5), 10021037. https://doi.org/10.1086/261420

Salinas, H., Albornoz, J., Reyes, A., Erazo, M. e Ide, R. (2006). Análisis de componentes principales aplicado a variables respecto a la mujer gestante en la Región de las Américas. Revista chilena de obstetricia y ginecología, 71(1), 17-25. https://doi.org/10.4067/S0717-75262006000100004

Sánchez, A. R. P. (2011). Desarrollo Económico Regional en España. Análisis de la productividad y del empleo como factores determinantes. Boletín económico de ICE, Información Comercial Española, (3007), 39-48.

Sánchez, F. y Nuñez, J. (2000). La geografía y el desarrollo económico en Colombia: una aproximación municipal. Desarrollo y sociedad, (46), 43-108. https://doi.org/10.13043/dys.46.2 
Smith, A. (1776). An Inquiry into the Nature and Causes of The Wealth of Nations. Edimburgo, Reino Unido: Stevenson \& co.

Strange, W. (2005). Urban Agglomeration. En Macmillan Publishers (Ed.), New Palgrave Dictionary of Economics, 2nd Edition. Nueva York, Estados Unidos: Macmillan.

UPME -Unidad de Planeación Minero Energética-. (2005). Atlas de Radiación solar de Colombia. Bogotá, Colombia: UPME, Ministerio de Minas y Energía.

Vázquez, A. (2000). Desarrollo endógeno y globalización. EURE (Santiago), 26(79), 47-65. https://doi. org/10.4067/S0250-71612000007900003

Villardón, J. (2002). Análisis de componentes principales. Barcelona, España: UOC.

Viloria, J. (2007). Economía del Departamento de Nariño: ruralidad y aislamiento geográfico. Cartagena, Colombia: Banco de la República. 